\title{
Large-scale genome-wide analysis identifies genetic variants associated with cardiac structure and function
}

Philipp S. Wild ${ }^{1,2,3}$ Janine F. Felix, ${ }^{4}$ Arne Schillert, ${ }^{5,6}$ Alexander Teumer, ${ }^{7,8}$ Ming-Huei Chen, ${ }^{9}$ Maarten J.C. Leening,${ }^{4,10}$ Uwe Völker, ${ }^{8,11}$ Vera Großmann, ${ }^{2}$ Jennifer A. Brody, ${ }^{12}$ Marguerite R. Irvin, ${ }^{13}$ Sanjiv J. Shah, ${ }^{14}$ Setia Pramana, ${ }^{15}$ Wolfgang Lieb, ${ }^{16}$ Reinhold Schmidt, ${ }^{17}$ Alice V. Stanton, ${ }^{18,19}$ Dörthe Malzahn, ${ }^{20}$ Albert Vernon Smith, ${ }^{21,22}$ Johan Sundström, ${ }^{23}$ Cosetta Minelli, ${ }^{24}$ Daniela Ruggiero, ${ }^{25}$ Leo-Pekka Lyytikäinen, ${ }^{26,27}$ Daniel Tiller, ${ }^{28}$ J. Gustav Smith, ${ }^{29,30,31}$ Claire Monnereau, ${ }^{4,32,33}$ Marco R. Di Tullio, ${ }^{34}$ Solomon K. Musani, ${ }^{35}$ Alanna C. Morrison, ${ }^{36}$ Tune H. Pers, ${ }^{37,38,39,40}$ Michael Morley, ${ }^{41}$ Marcus E. Kleber, ${ }^{42}$ AortaGen Consortium, ${ }^{43}$ Jayashri Aragam, ${ }^{44,45}$ Emelia J. Benjamin, ${ }^{46,47}$ Joshua C. Bis, ${ }^{12}$ Egbert Bisping, ${ }^{48}$ Ulrich Broeckel, ${ }^{49}$ CHARGE-Heart Failure Consortium, ${ }^{50}$ Susan Cheng, ${ }^{46,51}$ Jaap W. Deckers, ${ }^{10}$ Fabiola Del Greco $M,{ }^{52}$ Frank Edelmann, ${ }^{53}$ Myriam Fornage, ${ }^{54}$ Lude Franke,,${ }^{55}$ Nele Friedrich, ${ }^{8,56}$ Tamara B. Harris, ${ }^{57}$ Edith Hofer, ${ }^{17,58}$ Albert Hofman, ${ }^{4}$ Jie Huang, ${ }^{59,60}$ Alun D. Hughes, ${ }^{61}$ Mika Kähönen, ${ }^{62,63}$ KNHI investigators, ${ }^{64}$ Jochen Kruppa, ${ }^{5,65}$ Karl J. Lackner, ${ }^{66}$ Lars Lannfelt, ${ }^{67}$ Rafael Laskowski, ${ }^{68}$ Lenore J. Launer, ${ }^{69}$ Margrét Leosdottir, ${ }^{70}$ Honghuang Lin, ${ }^{46,71}$ Cecilia M. Lindgren, ${ }^{72,73}$ Christina Loley, ${ }^{5}$ Calum A. MacRae, ${ }^{73,74}$ Deborah Mascalzoni, ${ }^{52}$ Jamil Mayet, ${ }^{75,76}$ Daniel Medenwald, ${ }^{28}$ Andrew P. Morris, ${ }^{72,77}$ Christian Müller, ${ }^{78}$ Martina Müller-Nurasyid,,${ }^{79,80,81}$ Stefania Nappo, ${ }^{25}$ Peter M. Nilsson, ${ }^{82,83}$ Sebastian Nuding, ${ }^{84}$ Teresa Nutile, ${ }^{25}$ Annette Peters, ${ }^{80,85}$ Arne Pfeufer, ${ }^{86}$ Diana Pietzner, ${ }^{28}$ Peter P. Pramstaller, ${ }^{52,87,88}$ Olli T. Raitakari, ${ }^{89,90}$ Kenneth M. Rice, ${ }^{91}$ Fernando Rivadeneira, ${ }^{4,32,92}$ Jerome I. Rotter, ${ }^{93}$ Saku T. Ruohonen, ${ }^{90}$ Ralph L. Sacco,,${ }^{94,95,96}$ Tandaw E. Samdarshi, ${ }^{97}$ Helena Schmidt, ${ }^{98}$ Andrew S.P. Sharp, ${ }^{99}$ Denis C. Shields, ${ }^{100,101}$ Rossella Sorice, ${ }^{25,102}$ Nona Sotoodehnia, ${ }^{12,103}$ Bruno H. Stricker, ${ }^{4,92,104}$ Praveen Surendran, ${ }^{19,101}$ Simon Thom, ${ }^{75,76}$ Anna M. Töglhofer, ${ }^{98}$ André G. Uitterlinden, ${ }^{4,92}$ Rolf Wachter, ${ }^{105}$ Henry Völzke, ${ }^{7,8}$ Andreas Ziegler, ${ }^{5,6,106,107}$ Thomas Münzel, ${ }^{3,68}$ Winfried März, ${ }^{42,108,109}$ Thomas P. Cappola, ${ }^{41}$ Joel N. Hirschhorn, ${ }^{37,38,110}$ Gary F. Mitchell, ${ }^{111}$ Nicholas L. Smith, ${ }^{112,113,114}$ Ervin R. Fox, ${ }^{97}$ Nicole D. Dueker, ${ }^{115}$ Vincent W.V. Jaddoe, ${ }^{4,32,33}$ Olle Melander, ${ }^{82,83}$ Martin Russ, ${ }^{84,16}$ Terho Lehtimäki, ${ }^{26,27}$ Marina Ciullo,, ${ }^{25,102}$ Andrew A. Hicks, ${ }^{52}$ Lars Lind, ${ }^{23}$ Vilmundur Gudnason, ${ }^{21,22}$ Burkert Pieske, ${ }^{48,53,117}$ Anthony J. Barron, ${ }^{75,76}$ Robert Zweiker, ${ }^{48}$ Heribert Schunkert, ${ }^{80,118}$ Erik Ingelsson, ${ }^{119,120}$ Kiang Liu, ${ }^{14}$ Donna K. Arnett, ${ }^{13}$ Bruce M. Psaty, ${ }^{13,121}$ Stefan Blankenberg, ${ }^{6,78}$ Martin G. Larson, ${ }^{122,123}$ Stephan B. Felix, ${ }^{8,124}$ Oscar H. Franco, ${ }^{4}$ Tanja Zeller, ${ }^{6,78}$ Ramachandran S. Vasan, ${ }^{46,47}$ and Marcus Dörrr,124

'Preventive Cardiology and Preventive Medicine, Department of Medicine 2, and 2 'Center for Thrombosis and Hemostasis, University Medical Center of the Johannes Gutenberg-University Mainz, Mainz, Germany. ${ }^{3}$ DZHK (German Centre for Cardiovascular Research), partner site RhineMain, Mainz, Germany. ${ }^{4}$ Department of Epidemiology, Erasmus MC, University Medical Center Rotterdam, Rotterdam, Netherlands. Institute for Medical Biometry and Statistics, University Lübeck, University Medical Center Schleswig-Holstein, Lübeck, Germany. ${ }^{6}$ DZHK, partner site Hamburg/Kiel/Lübeck, Hamburg, Germany. Institute for Community Medicine, University Medicine Greifswald, Greifswald, Germany. ${ }^{8} \mathrm{DZHK}$, partner site Greifswald, Greifswald, Germany. ${ }^{9}$ Department of Neurology, Boston University School of Medicine, Boston, Massachusetts, USA. ${ }^{10}$ Department of Cardiology, Erasmus MC, University Medical Center Rotterdam, Rotterdam, Netherlands. "Interfaculty Institute of Cenetics and Functional Genomics, University Medicine Greifswald, Greifswald, Germany. ${ }^{2}$ Cardiovascular Health Research Unit, Department of Medicine, University of Washington, Seattle, Washington, USA. ${ }^{13}$ Department of Epidemiology, School of Public Health, University of Alabama at Birmingham, Birmingham, Alabama, USA. ${ }^{14}$ Northwestern University Feinberg School of Medicine, Chicago, Illinois, USA. ${ }^{15}$ Department of Medical Epidemiology and Biostatistics, Karolinska Institutet, Stockholm, Sweden. ${ }^{16}$ Institute of Epidemiology and Popgen Biobank, Christian-Albrechts University of Kiel, Kiel, Germany. ${ }^{17}$ Department of Neurology, Clinical Division of Neurogeriatrics, Medical University Graz, Graz, Austria. ${ }^{18}$ Blood Pressure Unit, Beaumont Hospital, Dublin, Ireland. ${ }^{19}$ Department of Molecular and Cellular Therapeutics, Royal College of Surgeons in Ireland, Dublin, Ireland. ${ }^{20}$ Department of Genetic Epidemiology, University Medical Center, Georg-August University, Göttingen, Germany. ${ }^{21}$ celandic Heart Association, Kopavogur, Iceland. ${ }^{22}$ Faculty of Medicine, University of Iceland, Reykjavik, Iceland. ${ }^{23}$ Department of Medical Sciences, Cardiovascular Epidemiology, Uppsala University, Uppsala, Sweden. ${ }^{24}$ Population Health and Occupational Disease, National Heart and Lung Institute (NHLI), Imperial College London, London, United Kingdom. ${ }^{25}$ Institute of Cenetics and Biophysics A. Buzzati-Traverso, CNR, Naples, Italy. ${ }^{26}$ Department of Clinical Chemistry, Fimlab Laboratories, Tampere, Finland. ${ }^{27}$ Department of Clinical Chemistry, Faculty of Medicine and Life Sciences, University of Tampere, Tampere, Finland. ${ }^{28}$ Institute of Medical Epidemiology, Biostatistics, and Informatics, Martin-Luther-University Halle-Wittenberg, Halle (Saale), Germany. ${ }^{29}$ Department of Cardiology, Lund University and Skåne University Hospital, Lund, Sweden. ${ }^{30}$ Program in Medical and Population Cenetics, Broad Institute, Cambridge, Massachusetts, USA. ${ }^{31}$ Center for Human Cenetic Research and Cardiovascular Research Center, Massachusetts General Hospital and Harvard Medical School, Boston, Massachusetts, USA. ${ }^{32}$ The Generation R Study Group and ${ }^{33}$ Department of Pediatrics, Erasmus MC, University Medical Center Rotterdam, Rotterdam, Netherlands. ${ }^{34}$ Department of Medicine, Columbia University Medical Center, New York, New York, USA. ${ }^{35}$ Jackson Heart Study, University of Mississippi Medical Center, Jackson, Mississippi, USA. ${ }^{36}$ Department of Epidemiology, Human Cenetics, and Environmental Sciences, University of Texas Health Science Center at Houston, Houston, Texas, USA. ${ }^{37}$ Medical and Population Cenetics Program, Broad Institute of MIT and Harvard, Cambridge, Massachusetts, USA. ${ }^{38}$ Division of Endocrinology and Center for Basic and Translational Obesity Research, Boston Children's Hospital, Boston, Massachusetts, USA. ${ }^{39}$ Novo Nordisk Foundation Center for Basic Metabolic Research, University of Copenhagen, Copenhagen, Denmark. ${ }^{40}$ Statens Serum Institut, Department of Epidemiology Research, Copenhagen, Denmark. ${ }^{41}$ Penn Cardiovascular Institute and Division of Cardiovascular Medicine, Perelman School of Medicine, University of Pennsylvania, Philadelphia, Pennsylvania, USA. ${ }^{42}$ Vth Department of Medicine, Medical Faculty Mannheim, Heidelberg University, Mannheim, Germany. ${ }^{43}$ Members of the AortaGen Consortium and their affiliations are detailed in the Supplemental Acknowledgments. ${ }^{44}$ Harvard Medical School, Boston, Massachusetts, USA. ${ }^{45}$ Veteran's Administration Hospital, West Roxbury, Boston, Massachusetts, USA. ${ }^{46}$ National Heart, Lung, and Blood Institute's and Boston University's Framingham Heart Study, Framingham, Massachusetts, USA. ${ }^{47}$ Sections of Cardiology, Preventive Medicine and Epidemiology, Department of Medicine, Boston University Schools of Medicine and Public Health, Boston, Massachusetts, USA. ${ }^{48}$ Department of Cardiology, Medical University Graz, Graz, Austria. ${ }^{49}$ Medical College of Wisconsin, Milwaukee, Wisconsin, USA. ${ }^{50}$ Members of the CHARGE-Heart Failure Consortium are detailed in the Supplemental Acknowledgments. ${ }^{51}$ Cardiovascular Division, Brigham and Women's Hospital, Harvard Medical School, Boston, Massachusetts, USA. ${ }^{52}$ Center for Biomedicine, European Academy of Bolzano/Bozen, Bolzano, Italy - Affiliated institute of the University of Lübeck, Lübeck, Germany. ${ }^{53}$ Department of Cardiology, Charité-Universitätsmedizin Berlin, Campus Virchow-Klinikum, Berlin, Germany. ${ }^{54}$ University of Texas Health Science Center, Houston, Texas, USA. ${ }^{55}$ Department of Genetics, University of Groningen, University Medical Centre Groningen, Groningen, Netherlands. ${ }^{56}$ Institute of Clinical Chemistry and Laboratory Medicine, University Medicine Greifswald, Greifswald, Germany. ${ }^{57}$ Laboratory of Epidemiology, Demography, and Biometry, National Institute on Aging, NIH, Bethesda, Maryland, USA. ${ }^{58}$ Institute for Medical Informatics, Statistics and Documentation, Medical University Graz, Graz, Austria. ${ }^{59}$ Boston VA Research Institute, Boston, Massachusetts, USA. ${ }^{60}$ Brigham and Women's Hospital Division of Aging, Harvard Medical School, Boston, Massachusetts, USA. ${ }^{61}$ nstitute of Cardiovascular Science, University College London, London, United Kingdom. ${ }^{62}$ Department of Clinical Physiology, Tampere University Hospital, Tampere, Finland. ${ }^{63}$ Department of Clinical Physiology, Faculty of Medicine and Life Sciences, University of Tampere, Tampere, Finland. ${ }^{44} \mathrm{KNHI}$ investigators 
and their affiliations are detailed in the Supplemental Acknowledgments. ${ }^{65}$ University of Veterinary Medicine, Foundation Institute of Veterinary Medicine and Genetics, Hannover, Germany. ${ }^{66}$ Institute of Clinical Chemistry and Laboratory Medicine, University Medical Center Mainz, Mainz, Germany. ${ }^{67}$ Department of Public Health and Caring Sciences, Geriatrics, Uppsala University, Uppsala, Sweden. ${ }^{68}$ Department of Medicine 2, University Medical Center Mainz, Mainz, Cermany. ${ }^{69}$ Neuroepidemiology Section, National Institute on Aging, NIH, Bethesda, Maryland, USA. ${ }^{70}$ Department of Cardiology, Lund University, and Skåne University Hospital, Malmö, Sweden. ${ }^{71}$ Section of Computational Biomedicine, Department of Medicine, Boston University School of Medicine, Boston, Massachusetts, USA. ${ }^{72}$ Wellcome Trust Centre for Human Cenetics, University of Oxford, Oxford, United Kingdom. ${ }^{73}$ Broad Institute of the Massachusetts Institute of Technology and Harvard University, Cambridge, Massachusetts, USA. ${ }^{74}$ Brigham and Women's Hospital, Boston, Massachusetts, USA. ${ }^{75}$ International Centre for Circulatory Health, Hammersmith Hospital, London, United Kingdom. ${ }^{76} \mathrm{NHLI}$, Imperial College London, London, United Kingdom. ${ }^{77}$ Department of Biostatistics, University of Liverpool, Liverpool, United Kingdom. ${ }^{78}$ Department of General and Interventional Cardiology, University Heart Center Hamburg, Hamburg, Germany. ${ }^{79}$ Department of Medicine I, Ludwig-Maximilians-University Munich, Munich, Germany. ${ }^{80} \mathrm{DZHK}$, partner site Munich Heart Alliance, Munich, Germany. ${ }^{81}$ Institute of Genetic Epidemiology, Helmholtz Zentrum München - German Research Center for Environmental Health, Neuherberg, Germany. ${ }^{82}$ Department of Clinical Sciences, Lund University, Malmö, Sweden. ${ }^{83}$ Department of Internal Medicine, Skåne University Hospital, Malmö, Sweden. ${ }^{84}$ Department of Medicine III, University Clinics Halle (Saale), Martin-Luther-University Halle-Wittenberg, Halle (Saale), Cermany. ${ }^{85}$ Institute of Epidemiology II, Helmholtz Zentrum München - German Research Center for Environmental Health, Neuherberg, Germany. ${ }^{86}$ Institute of Human Genetics, Helmholtz Zentrum München, Neuherberg, Cermany. ${ }^{87}$ Department of Neurology, General Central Hospital, Bolzano, Italy. ${ }^{88}$ Department of Neurology, University of Lübeck, Lübeck, Cermany. ${ }^{89}$ Department of Clinical Physiology and Nuclear Medicine, Turku University Hospital, Turku, Finland. ${ }^{90}$ Research Centre of Applied and Preventive Cardiovascular Medicine, University of Turku, Turku, Finland. ${ }^{91}$ Department of Biostatistics, University of Washington, Seattle, Washington, USA. ${ }^{92}$ Department of Internal Medicine, Erasmus MC, University Medical Center Rotterdam, Rotterdam, Netherlands. ${ }^{93}$ Institute for Translational Cenomics and Population Sciences, Los Angeles Biomedical Research Institute and Department of Pediatrics, Harbor-UCLA Medical Center, Torrance, California, USA. ${ }^{94}$ Department of Neurology and ${ }^{95}$ McKnight Brain Institute, Miller School of Medicine, University of Miami, Miami, Florida, USA. ${ }^{96}$ Departments of Public Health Sciences and Human Cenomics, University of Miami, Miami, Florida, USA. ${ }^{97}$ Division of Cardiology, University of Mississippi Medical Center, Jackson, Mississippi, USA. ${ }^{98}$ Institute of Molecular Biology and Biochemistry, Medical University Graz, Graz, Austria. ${ }^{99}$ Department of Cardiology, Royal Devon and Exeter Hospital and University of Exeter, Exeter, United Kingdom. ${ }^{100}$ UCD Conway Institute of Biomolecular and Biomedical Research and ${ }^{101}$ School of Medicine and Medical Sciences, University College Dublin, Dublin, Ireland. ${ }^{102}$ IRCCS Neuromed, Pozzilli, Isernia, Italy. ${ }^{103}$ Division of Cardiology, University of Washington, Seattle, Washington, USA. ${ }^{104}$ Inspectorate for Health Care, Utrecht, Netherlands. ${ }^{105}$ Department of Cardiology and Pneumology, University Medical Center of Cöttingen, Georg-August University, Göttingen, Germany. ${ }^{106}$ School of Mathematics, Statistics and Computer Science, University of KwaZulu-Natal, Durban, South Africa. ${ }^{107}$ Zentrum für Klinische Studien, Universität Lübeck, Lübeck, Germany. ${ }^{108}$ Synlab Academy, Synlab Services CmbH, Mannheim, Germany. ${ }^{109} \mathrm{Clinical}$ Institute of Medical and Chemical Laboratory Diagnostics, Medical University of Graz, Graz, Austria. ${ }^{110}$ Department of Cenetics, Harvard Medical School, Boston, Massachusetts, USA. ${ }^{11}$ Cardiovascular Engineering Inc., Norwood, Massachusetts, USA. ${ }^{12}$ Department of Epidemiology, University of Washington, Seattle, Washington, USA. ${ }^{113}$ Group Health Research Institute, Group Health Cooperative, Seattle, Washington, USA. ${ }^{114}$ Seattle Epidemiologic Research and Information Center, Department of Veterans Affairs Office of Research and Development, Seattle, Washington, USA. ${ }^{115}$ John P. Hussman Institute for Human Genomics, Miller School of Medicine, University of Miami, Miami, Florida, USA. ${ }^{116}$ Helios-Amperklinikum Dachau, Dachau, Cermany. ${ }^{117}$ Cerman Heart Institute Berlin DHZB, Department of Internal Medicine/Cardiology, Berlin, Germany. ${ }^{118}$ Deutsches Herzzentrum, Technische Universität München, Munich, Germany. ${ }^{119}$ Department of Medical Sciences, Molecular Epidemiology and Science for Life Laboratory, Uppsala University, Uppsala, Sweden. ${ }^{120}$ Department of Medicine, Division of Cardiovascular Medicine, Stanford University School of Medicine, Stanford, California, USA. ${ }^{121}$ Cardiovacular Health Research Unit, Departments of Medicine, Epidemiology, and Health Services, University of Washington, Seattle, Washington, USA. ${ }^{122}$ Biostatistics Department, Boston University School of Public Health, Boston, Massachusetts, USA. ${ }^{123}$ Department of Mathematics and Statistics, Boston University, Boston, Massachusetts, USA. ${ }^{124}$ Department of Internal Medicine B, University Medicine Greifswald, Greifswald, Germany.

BACKGROUND. Understanding the genetic architecture of cardiac structure and function may help to prevent and treat heart disease. This investigation sought to identify common genetic variations associated with inter-individual variability in cardiac structure and function.

METHODS. A GWAS meta-analysis of echocardiographic traits was performed, including 46,533 individuals from 30 studies (EchoGen consortium). The analysis included 16 traits of left ventricular (LV) structure, and systolic and diastolic function.

RESULTS. The discovery analysis included 21 cohorts for structural and systolic function traits $(n=32,212)$ and 17 cohorts for diastolic function traits $(n=21,852)$. Replication was performed in 5 cohorts $(n=14,321)$ and 6 cohorts $(n=16,308)$, respectively. Besides 5 previously reported loci, the combined meta-analysis identified 10 additional genome-wide significant SNPs: rs12541595 near MTSS1 and rs10774625 in ATXN2 for LV end-diastolic internal dimension; rs806322 near KCNRG, rs4765663 in CACNA1C, rs6702619 near PALMD, rs7127129 in TMEM16A, rs11207426 near FCGY, rs17608766 in COSR2, and rs17696696 in CFDP1 for aortic root diameter; and rs12440869 in IOCH for Doppler transmitral A-wave peak velocity. Findings were in part validated in other cohorts and in CWAS of related disease traits. The genetic loci showed associations with putative signaling pathways, and with gene expression in whole blood, monocytes, and myocardial tissue.

CONCLUSION. The additional genetic loci identified in this large meta-analysis of cardiac structure and function provide insights into the underlying genetic architecture of cardiac structure and warrant follow-up in future functional studies.

FUNDING. For detailed information per study, see Acknowledgments.

Authorship note: P.S. Wild, J.F. Felix, A. Schillert, and A. Teumer, as well as T. Zeller, R.S. Vasan, and M. Dörr, contributed equally to this work.

Conflict of interest: Author conflicts of interest are listed in the supplemental material. Submitted: October 5, 2015; Accepted: February 16, 2017.

Reference information: J Clin Invest. 2017;127(5):1798-1812.

https://doi.org/10.1172/JCl84840.

\section{Introduction}

Heart failure (HF) is associated with substantial morbidity, mortality, and health care costs, and is increasing in prevalence with the aging of the global population (1). Hence, prevention and treatment of $\mathrm{HF}$ by identifying its genetic and environmental determinants is a public health priority. The identification of the 


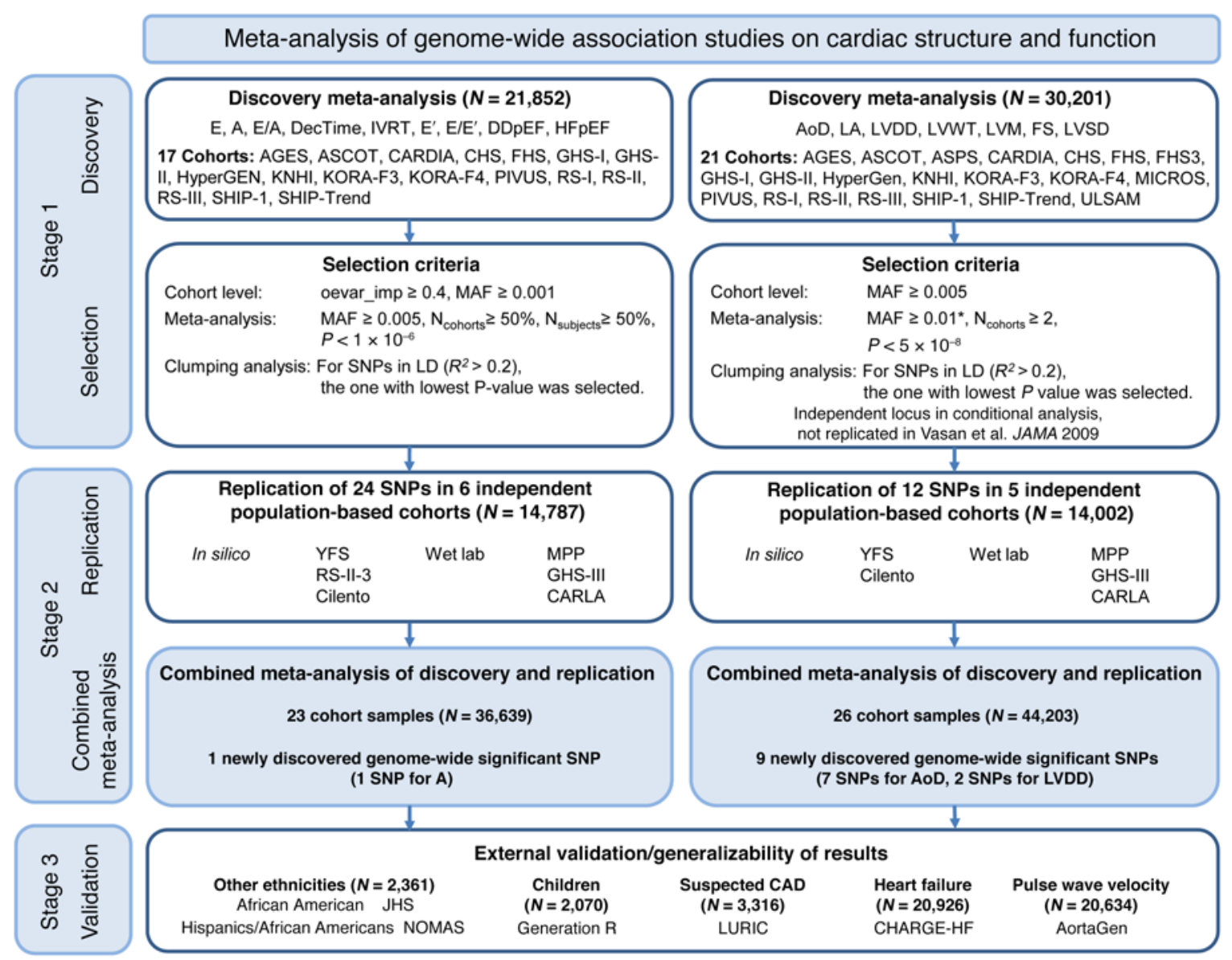

Figure 1. Flowchart of the analytical 3-stage approach. ${ }^{*}$ For LV systolic dysfunction as binary trait, the selection criterion for the MAF was $\geq 0.03$. Acronyms of cohorts are explained in the supplemental material. Mv-E (E), peak velocity of early diastolic transmitral inflow; Mv-A (A), peak velocity of transmitral inflow corresponding to atrial contraction; E/A, ratio of mitral E- and A-wave; DecTime, deceleration time of mitral E-wave; IVRT, isovolumetric relaxation time; $E^{\prime}$, peak velocity of excursion of lateral mitral annulus in early diastolic phase; $E / E^{\prime}$, ratio of $E$ and $E^{\prime}$; DDpEF, diastolic dysfunction with preserved ejection fraction; HFpEF, HF with preserved ejection fraction; LVM, LV mass; LVDD, LV diastolic dimension; LA, left atrium; FS, LV fractional shortening; LVSD, LV systolic dysfunction; MAF, minor allele frequency; $\mathrm{N}_{\text {cohorts }}$, number of cohorts included in analysis; $\mathrm{N}_{\text {subjects' }}$ number of subjects investigated per phenotype; LD, linkage disequilibrium; CAD, coronary artery disease; oevar_imp., observed divided by expected variance for imputed allele dosage. Vasan et al. JAMA 2009 is ref. 2.

genetic architecture of HF may be facilitated by evaluating echocardiographic traits of left ventricular (LV) structure and function. These heritable, quantitative traits can antedate HF and are more amenable to genetic analysis than more "distal" heart disease traits (2). Initial studies that related common genetic variants to echocardiographic traits and incident HF $(2-5)$ were limited by modest sample size, analysis of only a few echocardiographic phenotypes, or evaluation of "all HF," a phenotypically heterogeneous group (6-9).

We conducted a meta-analysis of genome-wide association studies (GWAS) on a comprehensive set of echocardiographic traits in carefully phenotyped individuals primarily of European ancestry within the EchoGen consortium (2) comprising 30 studies. We associated our identified genetic loci with echocardiographic traits in other ethnicities, in populations with related disease traits. Additionally, we further characterized loci by evaluating putative signaling pathways and examining their association with gene expression in whole blood, monocytes, and cardiac tissue.

\section{Results}

Cohort descriptions and the echocardiographic characteristics are presented in Supplemental Tables 1-5; supplemental material available online with this article; https://doi.org/10.1172/ JCI84840DS1.

Individual study genomic inflation factors are shown in Supplemental Table 6. The meta-analytic genomic inflation factor ( $\lambda$ ) was 1.09 or less for all traits evaluated. The genomic inflation factors for the traits with significant results (see below) were 1.09 (for aortic root diameter [AoD]) and 1.08 (for LV diastolic internal dimension [LVDD]). To address to what extent the genomic inflation might be due to unaccounted population stratification versus truly associated genetic markers, we applied the recently developed linkage disequilibrium (LD) score regression method to these two traits (10). The genomic inflation factor due to potential confounding bias reduced to 1.05 for AoD and to 1.03 for LVDD, suggesting that our meta-analytic approach accounted for population stratification reasonably well. Quantile-quantile (Q-Q) plots are shown in Supplemental Figures 1-16. 


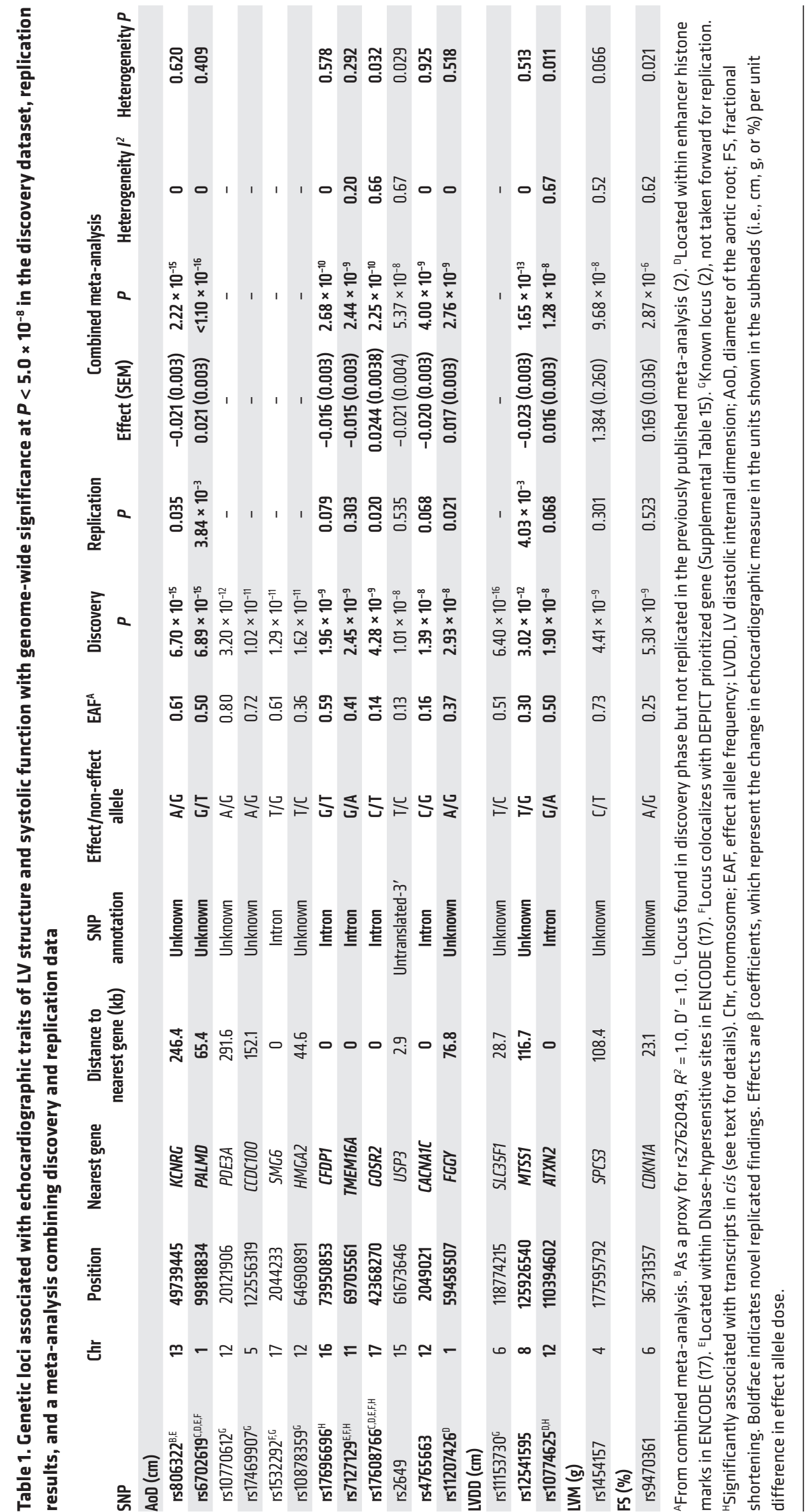


Single nucleotide polymorphisms related to cardiac structure and function (stage 1). We applied a two-stage design proposed by Skol et al. (11), including an additional stage for assessing the generalizability of the find, with details on samples and single nucleotide polymorphisms (SNPs) for each stage given in Figure 1. The meta-analysis of LV cardiac structure and systolic function traits included data from 21 cohorts with up to 30,201 individuals. For LV diastolic function, data were available from 17 cohorts with up to 21,852 individuals. We identified genome-wide significant associations (all $P<5 \times 10^{-8}$ ) of: 1 locus with LV mass (LVM), 3 with LVDD, 12 with AoD, 1 with LV fractional shortening (LVFS). Additionally, the following associations were observed at a higher $P$ value threshold (all $P<1 \times 10^{-6}$ ): 2 with the peak velocity of the transmitral E-wave (Mv-E), 5 with the peak velocity of the transmitral A-wave (Mv-A), 5 with the ratio of Mv-E to Mv-A (E/A), 2 with deceleration time of $\mathrm{Mv}-\mathrm{E}$ (DecTime), 4 with isovolumetric relaxation time (IVRT), 1 with the peak velocity of the excursion of the lateral mitral annulus in the early diastolic phase ( $\left.E^{\prime}\right), 3$ with the ratio of $\mathrm{Mv}-\mathrm{E}$ to $\mathrm{E}^{\prime}$ (E/E'), 1 with asymptomatic $\mathrm{LV}$ diastolic dysfunction with preserved ejection fraction (DDpEF), and 2 with $\mathrm{HF}$ with preserved ejection fraction ( $\mathrm{HFpEF})$. Using pre-defined selection criteria (Figure 1) and excluding known loci from our previous report (2), 12 SNPs for traits of cardiac structure and LV systolic function (Table 1) and 24 SNPs for traits of LV diastolic function (Table 2) were considered for additional analysis detailed in stage 2 below. Full results for all SNPs with $P<1 \times 10^{-4}$ are shown in Supplemental Table 7.

Replication and combined meta-analysis (stage 2). SNPs taken forward for stage 2 replication were analyzed in 5 cohorts $(n=$ 14,$002 ; 2$ with in silico GWAS data, 3 with de novo genotyping) for cardiac structure and LV systolic function; and in 6 cohorts ( $n=14,787 ; 3$ with in silico GWAS data, 3 with de novo genotyping) for LV diastolic function (Figure 1). A final combined meta-analysis of discovery and replication data from overall 30 cohort samples included 44,203 individuals with data on cardiac structure and systolic function, and 36,639 individuals with data on LV diastolic function. The investigation revealed 10 SNPs with genome-wide significance: rs10774625 and rs12541595 for LVDD; rs806322, rs4765663, rs6702619, rs7127129, rs11207426, rs17608766, and rs17696696 for AoD; and rs12440869 for Mv-A (Tables 1 and 2). Manhattan plots for these 3 traits are presented in Figure 2. Forest plots for the most significantly associated SNPs for AoD (rs6702619), LVDD (rs12541595), and Mv-A (rs12440869) with the corresponding regional plots including functional annotation are presented in Figures 3, 4, and 5. The plots for the other genome-wide significant loci are shown in Supplemental Figures 17 and 18. Funnel plots for the significantly associated SNPs are shown in Supplemental Figure 19. All known and novel loci combined explained $1.7 \%, 0.5 \%$, and $0.2 \%$ of the phenotypic variance in AoD, LVDD, and Mv-A, respectively, in a combined analysis of 3 of the larger cohorts.

Findings in children, other ethnicities, and related cardiovascular phenotypes (stage 3). In stage 3, the genome-wide significant SNPs were investigated for generalizability of the observed associations; small sample sizes of available cohorts partly limited the statistical power to replicate findings. In this exploratory analysis, we only found one weak association with AoD in white children of Europe- an ancestry in the Generation R study (12), and none in Hispanics (Northern Manhattan Study [NOMAS] study) or African Americans (Jackson Heart Study [JHS] and NOMAS study; Supplemental Table 8). When evaluating associations of the newly discovered SNPs with related disease traits, rs17696696, which was found to be associated with AoD, was also associated with pulse wave velocity in the AortaGen consortium (Supplemental Table 9 and ref. 13). There were no statistically significant associations with incident HF or mortality in HF patients of the CHARGE-Heart Failure (CHARGE-HF) consortium (Supplemental Table 10), or with all-cause mortality, HF, or cardiovascular mortality in the Ludwigshafen Risk and Cardiovascular Health (LURIC) cohort of patients with suspected coronary artery disease (CAD) (Supplemental Table 11). In the CARDIOGRAMplusC4D consortium data, rs17696696, rs17608766, and rs10774625 were significantly associated with CAD; rs10774625 was also strongly associated with the narrower phenotype myocardial infarction (MI; $P=$ $5.09 \times 10^{-11}$, Supplemental Table 12).

Biological pathways related to echocardiographic traits. In pathway analysis, the observed genetic variants were significantly enriched for canonical pathways that might be involved in the biological regulation of echocardiographic traits: protein kinase $\mathrm{A}$ signaling $\left(P=5.8 \times 10^{-6}\right)$, death receptor signaling $\left(P=6.9 \times 10^{-5}\right)$, the Wnt $/ \mathrm{Ca}^{2+}$ pathway $\left(P=2.2 \times 10^{-4}\right)$, and $\mathrm{P} 2 \mathrm{Y}$ purigenic receptor signaling $\left(P=4.1 \times 10^{-4}\right.$, Supplemental Tables 13 and 14, Supplemental Figure 20, and refs. 14-16).

When investigating the potential regulatory effect of the top loci using Encyclopedia of DNA Elements (ENCODE) data (17), 4 SNPs (rs10774625, rs6702619, rs17608766, and rs11207426) were located within enhancer histone marks and 4 (rs806322, rs6702619, rs7127129, and rs17608766) within DNase-hypersensitive sites. The literature search tool Snipper revealed no additional information, and no significant direct or indirect protein-protein interactions were found between loci using DAPPLE software (18). No significantly reconstituted gene sets were identified by the DEPICT tool (ref. 19 and Supplemental Table 15). DEPICT prioritized (false discovery rate $[\mathrm{FDR}]<0.05) 10$ genes across associated $\left(P<1 \times 10^{-5}\right)$ loci, including 4 colocalizing with genome-wide significant loci (Tables 1 and 2, and Supplemental Table 15).

Analyses of expression quantitative trait loci and gene expression in whole blood, monocytes, and myocardial tissue. Our data showed 4 SNPs that were significantly associated with cis transcripts in both datasets (whole blood and monocytes, Supplemental Table 16): rs10774625 with SH2B adaptor protein 3 ( $\mathrm{SH} 2 \mathrm{~B} 3, \mathrm{P}=8.15 \times 10^{-20}$ and $\left.P=1.83 \times 10^{-4}\right)$, rs17696696 with craniofacial development protein $1\left(C F D P 1, P=6.21 \times 10^{-11}\right.$ and $\left.P=7.59 \times 10^{-5}\right)$, rs7127129 with Fas-associated death domain-containing protein (FADD, $P=1.61 \times 10^{-37}$ and $\left.P=2.71 \times 10^{-4}\right)$, and rs1532292 with serine racemase $\left(S R R, P=3.40 \times 10^{-20}\right.$ and $\left.P=4.63 \times 10^{-10}\right)$.

We also examined the associations of our top loci with gene expression in human LV tissue provided by the Myocardial Applied Genomics Network consortium (MAGNet consortium; unpublished data). Two SNPs were significantly associated with LV gene expression: rs12541595 showed cis-association with metastasis suppressor 1 ( $M T S S 1, P=1.25 \times 10^{-19}$ ), with the effect allele T associated with lower MTSS1 expression; rs1532292 showed again a cis-association with $\operatorname{SRR}\left(P=2.62 \times 10^{-4}\right)$, with the effect allele $\mathrm{T}$ 


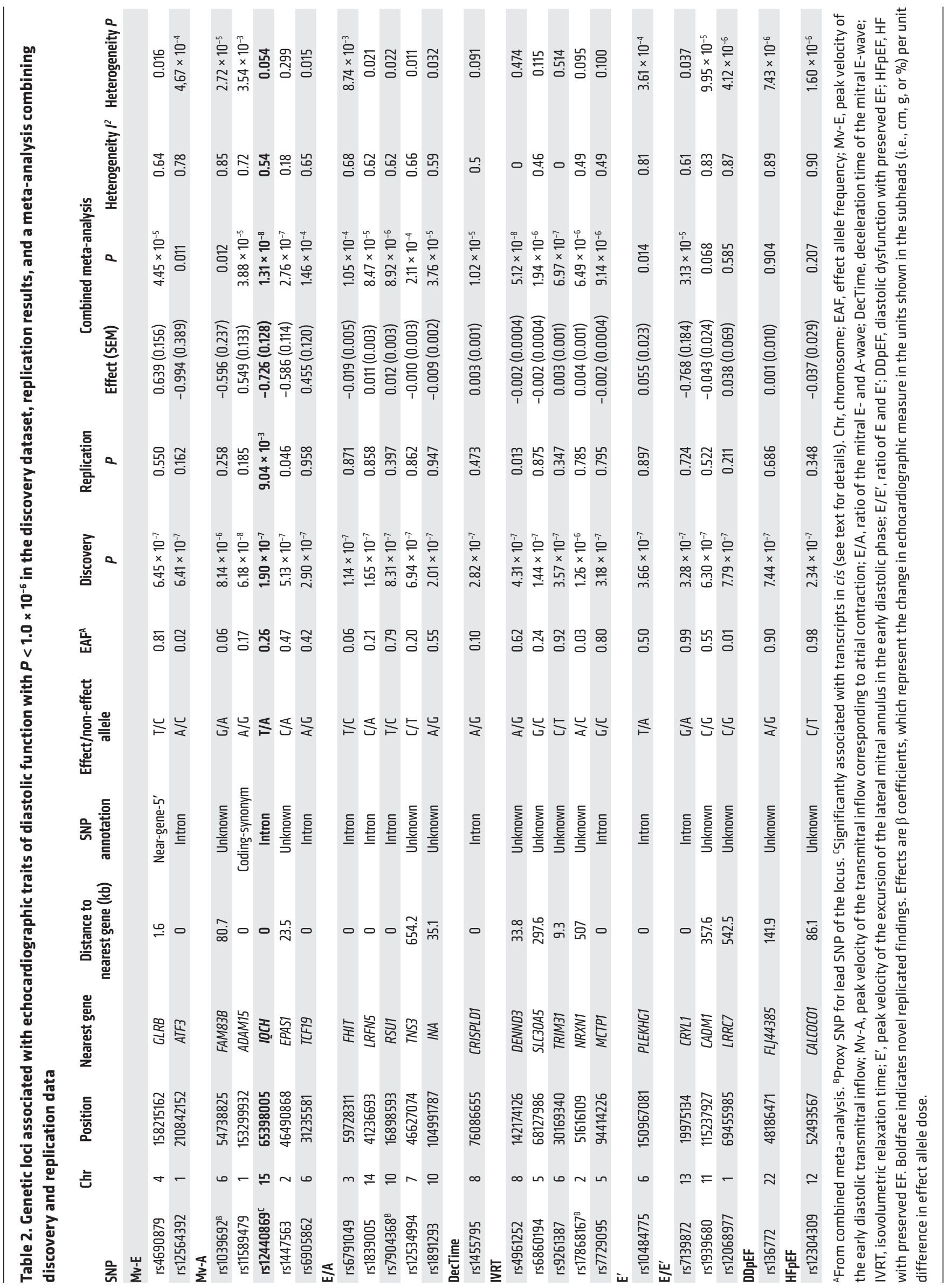


A

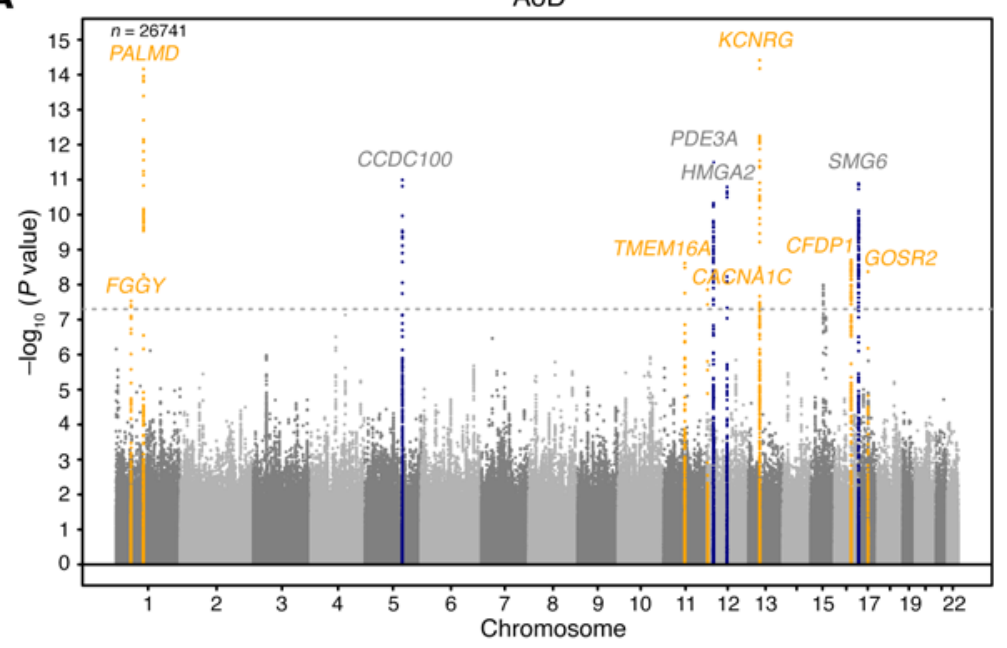

B

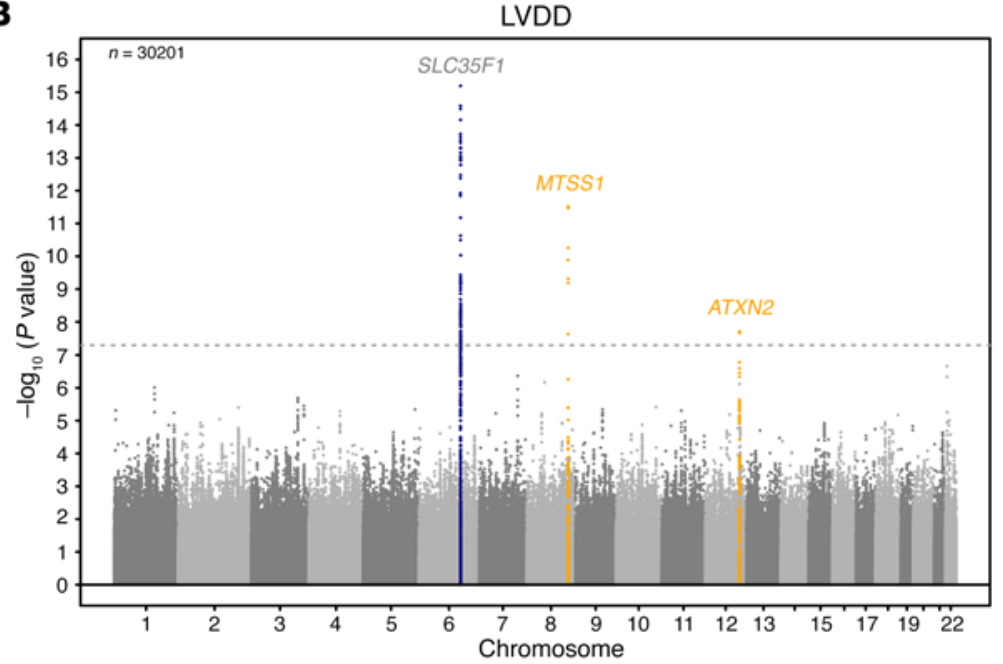

C

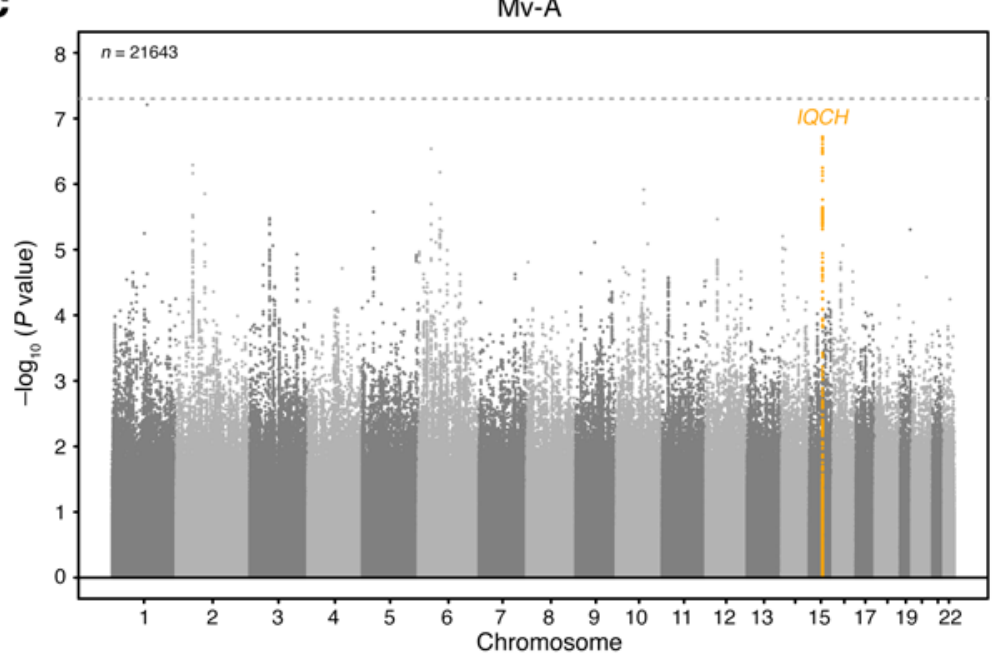

Figure 2. Manhattan plots of echocardiographic phenotypes with genome-wide findings in the joint analysis of discovery and replication cohorts. The plots show the SNP-wise log $P$ values against their genomic position for (A) aortic root diameter (AoD), (B) LV diastolic dimension (LVDD), and (C) peak velocity of the transmitral inflow corresponding to the atrial contraction (Mv-A). The genomewide significant loci and the gene nearest to the top SNP are highlighted in yellow if they were discovered in the present analysis and in blue if they had been identified in the earlier analysis. The horizontal gray lines indicate the significance threshold of $P=5 \times 10^{-8}$. $P$ values were obtained by calculating Wald test statistics. The number of samples ( $n$ ) per trait is reported in the upper left corner of each panel.

associated with lower gene expression levels, e.g., in the aorta and in blood cells. Additionally, the following eQTLs with genes from the reference sequence database (RefSeq; https://www.ncbi.nlm.nih.gov/ refseq/) in the aorta or heart tissue were found for the replicated SNPs in the GTEx database: rs17696696 (BCAR1), rs12541595 (LINC00964), and rs11153730 (SSXP10). Detailed GTEx results are given in Supplemental Table 17.

\section{Discussion}

In the present investigation, we identified 7 genetic loci associated with aortic root size and confirmed the associations of 4 other loci previously reported (2). These 11 variants explained $1.7 \%$ of the inter-individual variation in aortic root size (Supplemental Table 18). However, use of genome-wide complex trait analysis (GCTA) software in one of the larger cohorts (Study of Health in Pomerania [SHIP]) as an illustrative example demonstrated that common genetic variation explains about $30 \%$ of the variation in $\mathrm{AoD}$ (Supplemental Table 19), underscoring the potential for more, as-yet-undiscovered, loci. Additionally, we observed three genetic loci that were associated with LV diastolic dimensions (including one previously reported; see below) and one locus that was associated with the transmitral A-wave velocity.

Among the SNPs identified in our study as being associated with LVDD, one was rs12541595 close to MTSS1, which interacts with cytoplasmic actin near the cell surface and modulates intercellular connections in the kidney and metastatic potential in tumors $(20,21)$. When investigating our top loci for cisassociations with gene expression in human LV myocardial tissue (MAGNet consortium, unpublished data) and the GTEx database, rs12541595 showed a significant association with MTSS1 expression, with the LVDD-lowering allele (T) associated with lower associated with lower $S R R$ expression. Both expression quantitative trait locus (eQTL) associations from the LV tissue were also supported by the GTEx database (http://gtexportal.org/home/). The association with SRR expression for rs1532292 had the same direction of effect in different tissues, with the $\mathrm{T}$ allele generally MTSS1 expression in this tissue (Supplemental Table 9). We speculate that a reduction in MTSS1 may promote favorable LV remodeling, perhaps by affecting cell junctions. The other novel variant associated with LVDD, rs10774625, was associated with expression of SH2B3 in eQTL analysis and lies in ATXN2 (ataxin 2), 


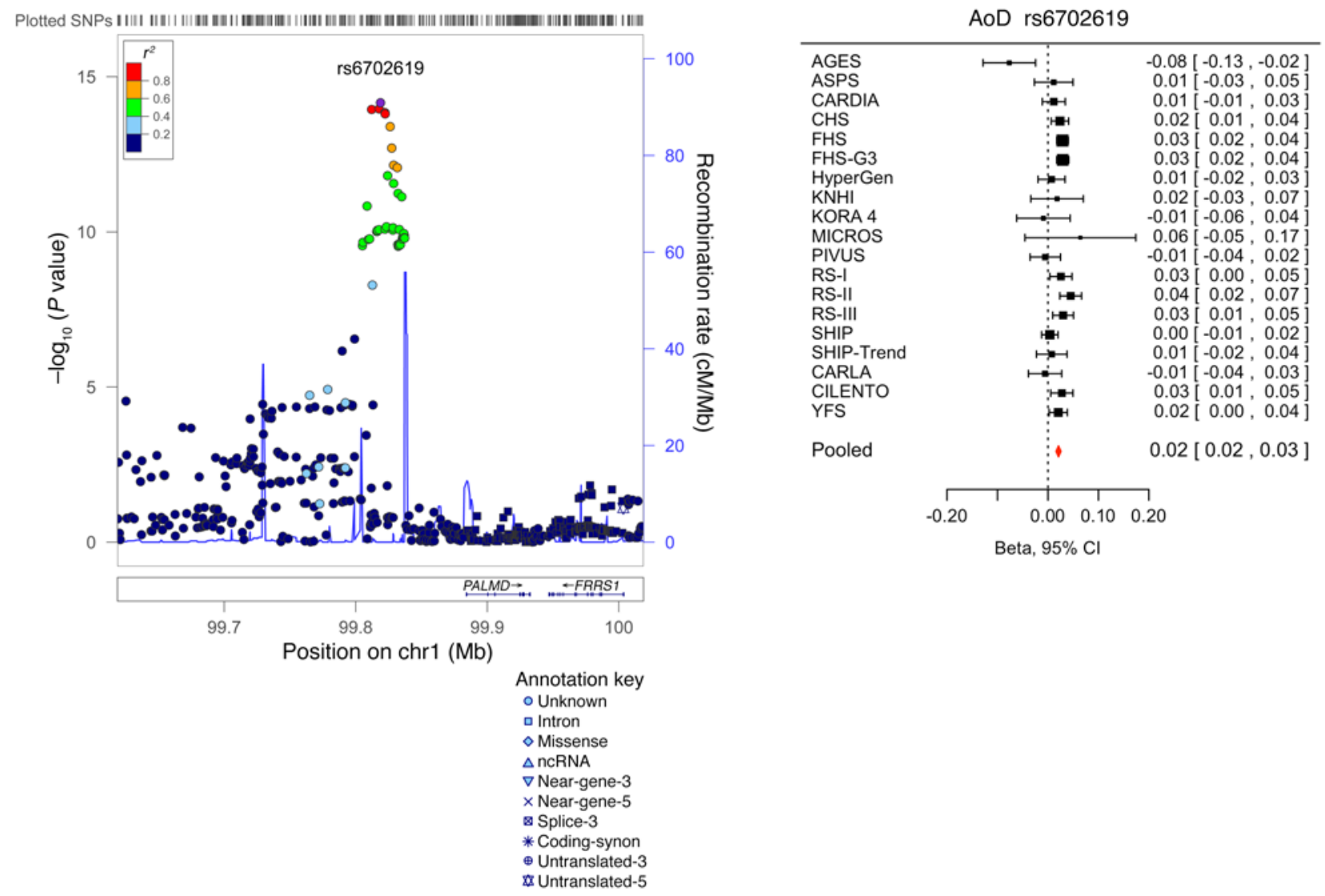

Figure 3. Forest plot for the meta-analysis of the association between rs6702619 and AoD, with the corresponding regional plot including functional annotation. $P$ values were obtained by calculating Wald test statistics using a sample size of $n=26,741$. Total sample size in the forest plot is $n=30,704$.

which is adjacent to $S H 2 B 3$, previously associated with retinal venular diameter, CAD, and arterial hypertension in separate reports (22-26). For LVDD, we also replicated the previously identified SLC35F1 locus (soluble transporter membrane protein) adjacent to the phospholamban (PLN) locus (protein inhibiting cardiac muscle sarcoplasmic reticulum $\mathrm{Ca}^{++}$-ATPase) (2).

Three loci associated with AoD have been linked previously to blood pressure as well as MI (GOSR2, Golgi SNAP receptor complex member 2; refs. 24, 27), blood pressure response to treatment $(C A C N A 1 C$, calcium channel, voltage-dependent, L type, alpha 1C subunit; ref. 28), and carotid intimal-medial thickness, as well as with CAD (CFDP1; refs. 29, 30). The other novel AoD-associated genetic loci were in or close to PALMD (palmdelphin, a paralemmin-related cytosolic protein; ref. 31), KCNRG (soluble protein with regulatory function in voltagegated potassium channels; ref. 32), FGGY (carbohydrate kinase domain-containing protein, phosphorylates carbohydrates; ref. 33), and in TMEM16A (transmembrane member 16A, protein involved in transepithelial anion transport and smooth muscle contraction; ref. 34). We also replicated in our discovery sample 4 loci associated with aortic diameter from our previous report (2): SMG6 (Smg-6 homolog, nonsense-mediated mRNA decay factor), CCDC10O (centrosomal protein $120 \mathrm{kDa}$ ), HMGA2 (high-mobility group AT-hook 2), and PDE3A (phosphodiesterase 3A, cGMP-inhibited). The effect allele of rs1532292 was associated with lower SRR expression in human LV myocardial tissue (unpublished data from the MAGNet consortium; GTEx database, see Supplemental Table 9).

One of the SNPs associated with AoD in our meta-analysis was also associated with AoD in children in the Generation R Study. Additionally, one SNP was associated with pulse wave velocity. Two SNPs associated with AoD and one SNP associated with LVDD were also significantly associated with CAD, the LVDD SNP also with MI in the CARDIOGRAMplusC4D consortium. These associations strengthen the evidence of involvement of these loci in echocardiographic traits. However, given the sample sizes of cohorts with different ethnicities as well as the SNP allele frequencies, and taking the effect sizes into account, the power was not more than $35 \%$ to reveal a statistically significant association of select SNPs with traits in "look-up" exercises. Therefore, some of the null results for the assessment of the generalizability of observed associations to non-European samples should be interpreted with care.

Pathway analysis suggested enrichment of the Wnt $/ \mathrm{Ca}^{2+}$ canonical pathway among the genetic variants associated with echocardiographic traits. These observations are consistent with the known effects of this pathway on myocardial biology (35). The Wnt $/ \mathrm{Ca}^{2+}$ pathway connects to the nuclear factor of activated $\mathrm{T}$ cells (NFAT) transcription factor $(14,15)$ and gene expression via calcineurin. Interestingly, both calcineurin and its target NFAT are involved in cardiac hypertrophy (16).

The association of our findings with expression data from human blood revealed 4 genes with potential functional signif- 


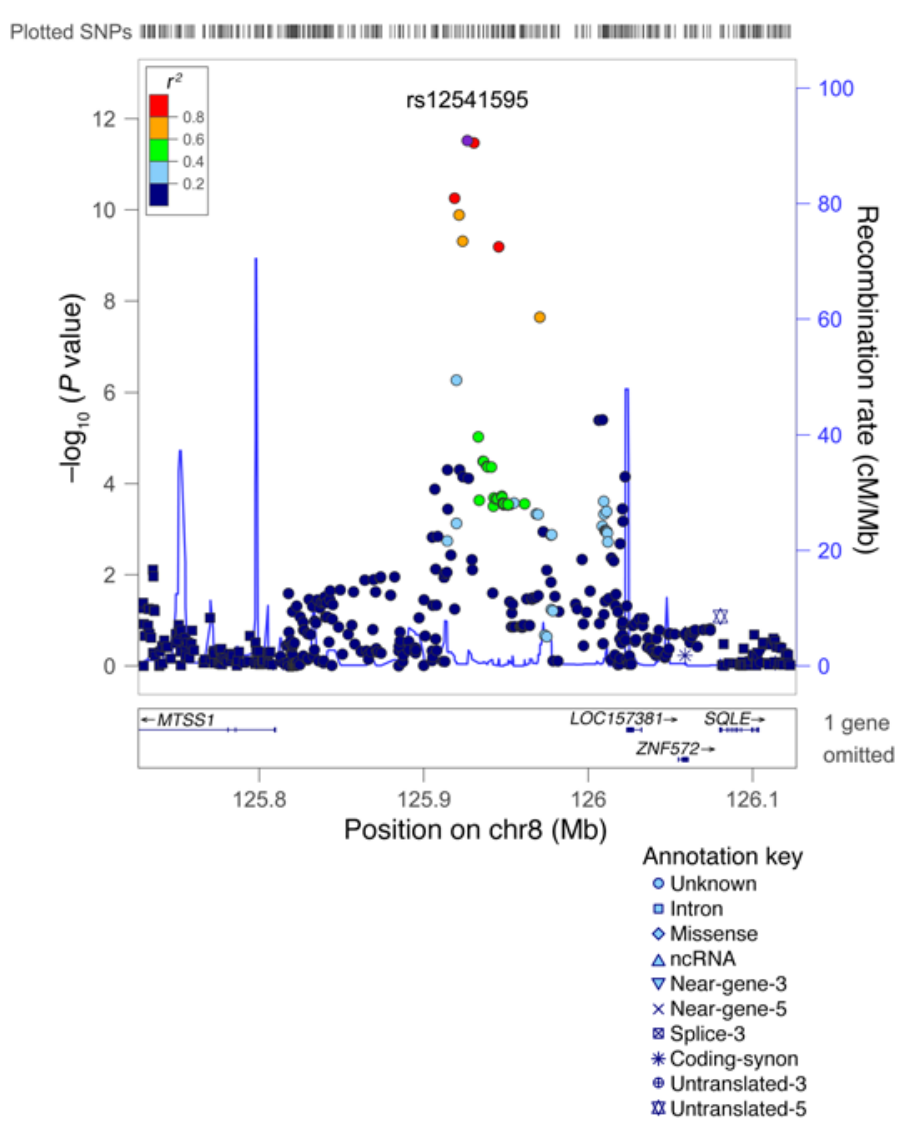

LVDD rs12541595

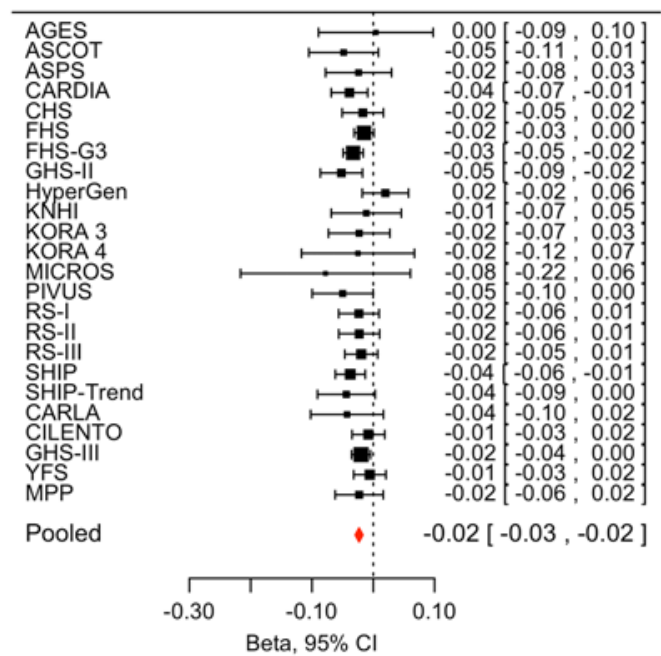

Figure 4. Forest plot for the meta-analysis of the association between rs12541595 and LVDD, with the corresponding regional plot including functional annotation. $P$ values were obtained by calculating Wald test statistics using a sample size of $n=30,201$. Total sample size in the forest plot is $n=43,623$.

icance (Supplemental Table 8). Of these, rs7127129 is located within TMEM16A, but its eQTL FADD has been shown to be associated with myocardial ischemia/reperfusion injury in an HF mouse model (36).

Our study is strengthened by the large sample size, the use of standardized echocardiographic techniques with adequate quality, and a harmonization of phenotypic data. Nonetheless, several limitations must be acknowledged. We did not observe any association of common genetic variants with the other echocardiographic measurements studied, e.g., LA size, LV wall thickness (LVWT), LVM, LV systolic dysfunction (LVSD), and most measures of LV diastolic function, with the exception of the transmitral A-wave velocity. In particular, we did not find any statistically significant associations for HFpEF, although we included only carefully phenotyped individuals in our study to reduce the phenotypic heterogeneity (37). The lack of association of select echocardiographic traits with common genetic variation is intriguing. It is likely that heterogeneity in both phenotypic assessment and study design and modest statistical power may have limited our ability to detect modest genetic associations, and associations with rare variants could not be assessed by design. A proportion of the intra-individual variability of functional traits might have been influenced by physiological factors (e.g., posture, state of hydration, heart rate, or medication use) (38). In this context, it should be noted that some echocardiographic measures may be imprecise, e.g., analysis of tissue Doppler imaging (TDI) of the mitral annulus would likely have further improved diagnosis and classification of LV diastolic dysfunction in our study if this method had been available in more cohorts. Likewise, as noted above, several of the LV diastolic filling measures are notoriously susceptible to variation in ventricular loading conditions (38). The genetic variants identified in our study have small effect sizes and explain a relatively small percentage of the variance in the echocardiographic phenotypes. Larger studies with more detailed reference panels, as well as more detailed functional studies and studies into the interactions of the variants found with factors such as hypertension, will likely shed further light on the molecular mechanisms underlying these complex traits. Furthermore, alterations of the transmitral A wave velocity are challenging to interpret alone, without consideration of other measures of LV diastolic function and filling patterns. The transmitral A wave velocity reflects the late diastolic phase of the LV filling, i.e., the phase of atrial contraction. Thus, in theory this single measure provides important information about active atrial function. Yet in practice, this measure changes variably and in a complex manner with the progression of LV diastolic dysfunction: Increasing impaired ventricular relaxation is at first accompanied by a decrease in E-wave with a compensatory increase in A-wave, resulting in a "relaxation abnormality" pattern; it results in the further, continuous decrease in A-wave velocity, reflecting a progressive deterioration of the contractility of the left atrium, and also changes in LV compliance $(39,40)$. These pathophysiological considerations underline the importance of the active contraction 


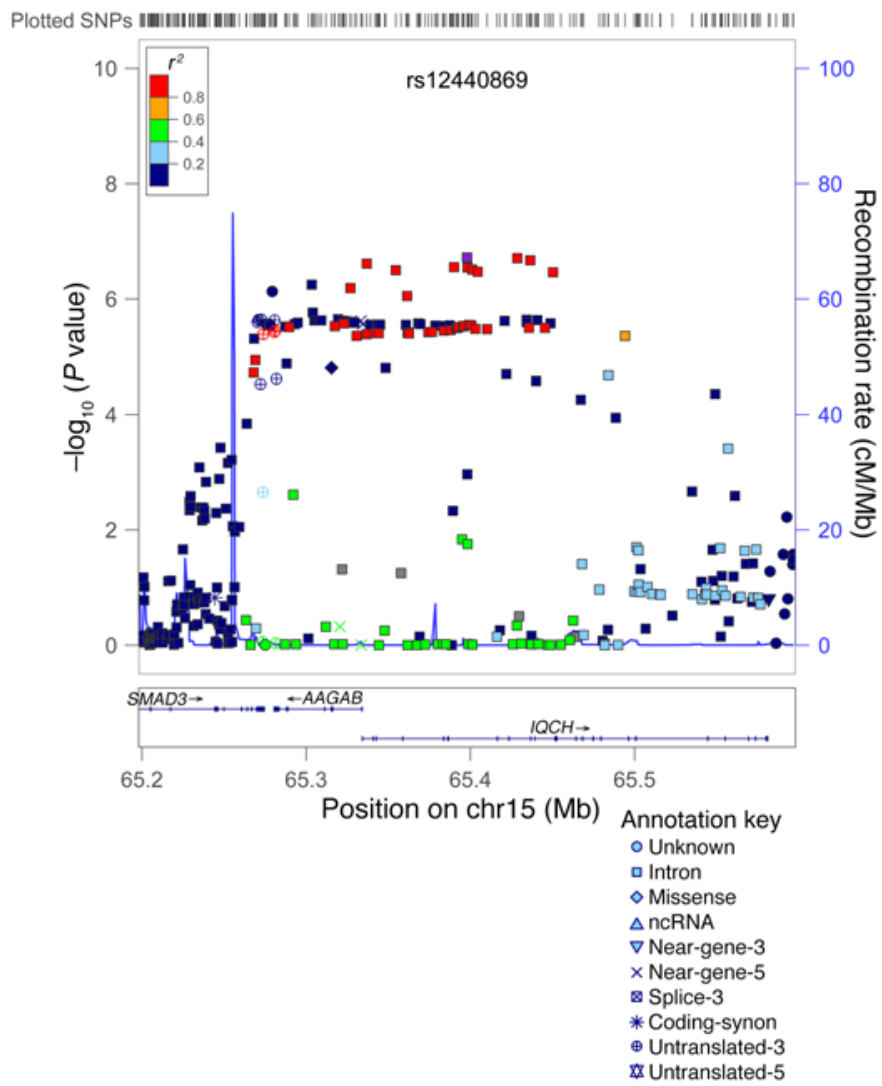

Mv-A rs12440869

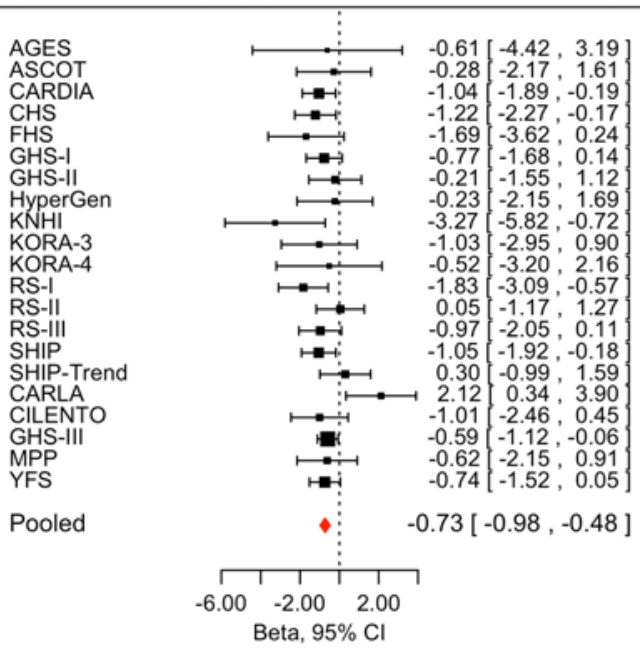

Figure 5. Forest plot for the meta-analysis of the association between rs12440869 and Mv-A, with the corresponding regional plot including functional annotation. $P$ values were obtained by calculating Wald test statistics using a sample size of $n=21,156$. Total sample size in the forest plot is $n=36,430$.

of the left atrium. Last, we did not directly assess the functional significance of all the associated SNPs or perform mechanistic studies, other than for the MTSS1 locus associated with LVDD (unpublished data from the MAGNet consortium).

To conclude, we report the largest genetic association study to our knowledge of a comprehensive set of LV echocardiographic traits. The large number of interesting genetic loci identified for AoD and LV diastolic dimensions, and the biological pathways enriched within our association results provide new insights into the biology of cardiac remodeling. Additional studies are warranted to further evaluate experimentally the functional significance of the reported genetic variants and loci.

\section{Methods}

\section{EchoGen consortium}

The EchoGen consortium was initiated in 2007 and has grown to a consortium of 30 studies with population-based and hospital-based cohorts primarily of European ancestry, and additionally including two cohorts of African American and one of Hispanic individuals. For the present investigation, we applied harmonized phenotype definitions, covariate selection, and genotyping protocols and the same statistical analysis plan across all cohorts. For traits of cardiac structure and systolic function, individuals with a history of MI, clinical diagnoses of $\mathrm{HF}$, or valve disease were excluded if this information was known or recorded during the echocardiographic examination.
For analysis of LV diastolic dysfunction, we excluded individuals with reduced ejection fraction (EF) (defined as $<50 \%, \mathrm{LVFS}<29 \%$ or poor/ impaired LV systolic function by visual estimation).

\section{Strategy for analysis}

For the identification of genetic variants associated with cardiac structure and function, we followed a 3-stage analysis plan (Figure 1). First, a discovery meta-analysis of up to 21 population- and hospital-based GWAS was performed (stage 1). Second, replication of the findings from stage 1 was performed in up to 6 independent cohort studies (3 with in silico data and 3 with de novo genotyping), and a combined meta-analysis of discovery and replication data was carried out (stage 2). In stage 3 , SNPs that were genome-wide significant in the combined meta-analysis were investigated for the generalizability of the observed associations in a cohort of white children of European ancestry (the Generation R study), in two cohorts of other ethnicities (Hispanic in the NOMAS Study and African American in the JHS and in the NOMAS study), and in associations with related disease traits (data from the AortaGen and CHARGE-HF consortia, and the LURIC study).

\section{Echocardiographic methods}

Detailed echocardiographic methods used and distributions of traits in each cohort study are reported in Supplemental Methods and Supplemental Tables 3 and 4 .

The present investigation focused on 5 traits of cardiac structure: LVM, LVDD, LVWT, AoD, and left atrial size (LA). Additionally, 
we evaluated 2 traits of systolic cardiac function (LVFS and LVSD) and 9 traits of $\mathrm{LV}$ diastolic function: $\mathrm{Mv}-\mathrm{A}, \mathrm{Mv}-\mathrm{E}, \mathrm{E} / \mathrm{A}, \mathrm{E}^{\prime}$, the ratio $\mathrm{E} / \mathrm{E}^{\prime}$ as a surrogate for LV end-diastolic pressure, DecTime, and IVRT, as well as DDpEF and HFpEF (41). Measurements were based on the European and American guidelines for the echocardiographic assessment of the LV (42).

\section{Genotyping methods and imputation}

Details on genotyping, imputation, and quality control are presented in Supplemental Table 5. Population stratification as well as family structure, if applicable, was accounted for in each individual cohort's analysis. For replication, 3 of the 6 cohorts (Gutenberg Health Study III [GHS-III]; Cardiovascular Risk Factors, Living and Ageing in Halle [CARLA] study; and Malmö Preventive Project [MPP] study) underwent de novo genotyping using $5^{\prime}$ nuclease assays on 384-well plates. For quality control, genotypes were validated in $10 \%$ of the samples for all SNPs.

\section{Definition of traits and statistical methods}

Discovery (stage 1). All traits were analyzed as continuous traits, with the exception of LVSD, DDpEF, and HFpEF. LVSD was defined as an $\mathrm{EF}<50 \%$, fractional shortening (FS) $<29 \%$ or reduced (poor or impaired) EF by visual estimation. Aggregate binary phenotypes were defined for asymptomatic participants with echocardiographic evidence of LV DDpEF and for those with HFpEF based on information on classes of HF according to the New York Heart Association (NYHA) and medication for HF in addition to echocardiography.

Stage 1 analyses were first performed separately at the individual cohort level for each trait (Figure 1). Continuous echocardiographic traits were related to genotype dosage ( $0-2$ copies of the effect allele) for each autosomal SNP using linear regression assuming additive genetic models adjusted for age, sex, height, weight, and study site (only applicable for the Cardiovascular Health Study [CHS] and Anglo-Scandinavian Cardiac Outcomes Trial [ASCOT]). For binary traits, we used logistic regression models with the same adjustments. In the Framingham Heart Study (FHS), linear mixed-effects models were applied to account for familial correlations. The associations of genotypes with echocardiographic traits were quantified by beta estimates, SEM, and $P$ values. After verifying strand alignment across studies and applying genomic control to each study, we performed inverse variance-weighted fixed-effects meta-analysis across the discovery cohorts with METAL (43) for the structural and the systolic function traits and with the R package MetABEL (http:// www.r-project.org) for the diastolic traits. After the meta-analysis, we excluded SNPs with a minor allele frequency (MAF) below $0.5 \%$ for diastolic function traits and below $1 \%$ for structural traits, and FS and below $3 \%$ for LVSD.

We used an a priori $P$ value threshold of $<5 \times 10^{-8}$ to indicate genome-wide statistical significance in the discovery meta-analysis for the selection of SNPs taken forward to the next stage. As no SNP reached genome-wide significance in the analysis of diastolic function traits, SNPs with $P<1 \times 10^{-6}$ were taken forward for replication as "suggestive" findings. This threshold was chosen because there was approximately $80 \%$ power to achieve a genome-wide significant $P$ value in the combined discovery and replication analysis for most of the traits given the effect sizes observed in the discovery stage. The association results were grouped based on the LD structure from the Hap-
Map (https://www.genome.gov/10001688/international-hapmapproject/) release $28 \mathrm{CEU}$ dataset using PLINK (settings $r^{2}>0.2,1 \mathrm{Mb}$ distance) (44). For each identified independent locus, the SNP with the lowest $P$ value was defined as the lead SNP and taken forward for replication. SNPs representing loci identified and replicated in our previously published report (2) were not taken forward for replication.

Replication and combined meta-analysis (stage 2). In stage 2, SNPs were related to echocardiographic traits in 6 cohort samples (Figure 1). We chose proxies for 4 of the top SNPs, as no reliable assays were available for wet lab replication of the originally identified SNPs: rs1039692 was used as a proxy for rs949796 (Mv-A, $P=6.60 \times 10^{-7}$, $\left.R^{2}=1.0\right)$, rs7904368 as a proxy for rs7074647 (E/A, $P=8.30 \times 10^{-7}$, $R^{2}=0.95$ ), rs17868167 as a proxy for rs17862703 (IVRT, $P=9.70 \times 10^{-7}$, $R^{2}=1.0$ ), and rs806322 as a proxy for rs 2762049 (AoD, $P=3.85 \times 10^{-15}$, $R^{2}=1.0$ ). The dbSNP (https://www.ncbi.nlm.nih.gov/projects/SNP/) identifiers of the proxies are reported in the final results.

For the combined meta-analysis of discovery and replication cohorts, SNPs with a $P$ value of $<5 \times 10^{-8}$ in the combined metaanalysis were considered to be significantly associated with their respective outcomes, as the overall sample size of the replication cohorts was very small. Genome-wide significant association signals were deemed novel for the corresponding traits if they were $>500 \mathrm{~kb}$ away from the lead SNPs reported in our previous study (2) and not in high $\mathrm{LD}$ with them $\left(R^{2}<0.5\right)$.

\section{Look-up in other cohorts to test for generalizability of findings}

For the genome-wide significant SNPs representing novel loci, we performed "look-ups" in relation to the corresponding echocardiographic traits in children (the Generation R study), Hispanics (NOMAS), and African Americans (meta-analysis of data from JHS and NOMAS). Additionally, we evaluated associations of these SNPs with traits of interest: SNPs for aortic root diameter with pulse wave velocity in the AortaGen consortium $(45,46)$; and all newly identified SNPs with incident HF and mortality in the CHARGE-HF consortium (3), with all-cause, cardiovascular, and HF mortality in the LURIC study (a cohort of patients with suspected CAD), as well as with MI and CAD in the CARDIOGRAMplusC4D consortium data (47). Further details for the look-up investigations are presented in Supplemental Methods.

\section{Proportion of trait variance explained}

The proportion of variance in echocardiographic traits explained by the significantly associated SNPs from our meta-analyses was estimated in 3 of the larger cohorts (Rotterdam study [RS], SHIP, and FHS). Within each cohort, $R^{2}$ values of two models were compared for each trait: one model including the covariates (age, sex, height, and weight) only; and one model additionally including the new and known loci. The proportion of the sex-, age-, height-, and weight-adjusted variance explained by all common (MAF >0.01) autosomal genotyped SNPs for each trait was calculated in the SHIP sample using the REML method of GCTA software version 1.24.4 (48).

\section{Known associations of genome-wide significant SNPs}

We combined a manual review of the literature with the use of the tool Snipperversion 1.2 (http://csg.sph.umich.edu/boehnke/snipper/), which conducts an automated search of the published literature using specified search terms and the putative SNP to evaluate previously reported disease/trait associations for the genome-wide significant SNPs. 


\section{cis eQTL analysis}

To evaluate the potential functional significance of our findings, we related each replicated SNP to the expression levels of genes in three sets of tissues: human whole blood samples from $n=5,311$ individuals evaluated by Westra et al. (49), human monocytes from $n=1,372$ participants in the GHS (50), and LV free-wall tissue from $n=313$ patients with $\mathrm{HF}$ undergoing transplantation and from unused donor hearts from the MAGNet consortium (http://www.med.upenn.edu/magnet). Further details are presented in Supplemental Methods. To evaluate possible cis eQTLs across multiple tissues, an additional look-up was performed in the GTEx database for the new findings.

\section{Pathway analysis}

The collective effects of multiple genetic variants on biological systems were investigated by pathway analysis, first for the 7 structural and systolic traits combined, and then for the 9 combined diastolic traits and for all 16 echocardiographic traits combined (for details, see Supplemental Methods).

To identify whether any of the associated SNPs fall within regulatory regions of the genome, we evaluated data from ENCODE (17). We compared the expected overlap of the putative SNPs with functional domains due to chance with the actual observed overlap by creating a permuted set of non-associated SNPs that were evaluated for overlap with the functional domains. We also used the DEPICT tool to further explore functionality of the identified SNPs (19). In addition, variants with $P<5 \times 10^{-7}$ were used as the input for the DAPPLE software (18), which then built both direct and indirect interaction networks from seed genes near the top loci.

\section{Statistics}

If not specified otherwise, a Wald test statistic was calculated by dividing the estimated effect size by its standard error and comparing them with a normal distribution (2-tailed) with mean zero. In the GWAS, $P<5 \times 10^{-8}$ for the combined stage 1 and 2 analysis was deemed significant (11), which corresponds to a significance level of 0.05 after correcting for 1 million independent SNPs (51). For pathway analyses, a FDR was applied as multiple testing correction with a cutoff-value $<0.05$ for statistical significance.

\section{Study approval}

All study protocols of participating cohorts were reviewed and approved by a local ethics committee and followed the recommendations of the Declaration of Helsinki. All subjects in the cohorts provided informed written consent prior to their participation in the study. Therefore, no specific approval was required for this meta-analysis of human data. The institutional review boards are listed in the supplemental material.

\section{Author contributions}

Conception and study design: AH, A. Peters, AS, AZ, BMP, BP, DT, EB, EI, GFM, H. Schmidt, H. Schunkert, JFF, LJL, LK, MD, MF, MK, OTR, PPP, PSW, R. Schmidt, RZ, SB, SBF, SJS, TBH, TL, TM, TZ, RSV, V. Gudnason, and WM. Sample collection and phenotyping: ADH, AJB, A. Peters, APM, ASPS, A.V. Smith, A.V. Stanton, BMP, CML, DCS, DKA, D. Mascalzoni, DR, DT, EB, EI, EJB, ERF, FE, FN, GFM, H. Schunkert, HV, JA, JM, JS, JWD, KJL, KL, LJL, L. Lannfelt, L. Lind, MC, MD, MGL, MJGL, MK, ML, MR, MRDT, NF, NLS,
OTR, PMN, PS, PSW, RL, RLS, RM, R. Schmidt, R. Sorice, RSV, RW, RZ, SB, SC, SJS, SKM, S. Nappo, ST, STR, TBH, TES, TL, TM, TN, TZ, V. Großmann, and V. Gudnason. Genotyping: AAH, AGU, AMT, A. Pfeufer, APM, CML, DR, EI, ERF, FR, H. Schmidt, JGS, JH, JIR, JS, KJL, KL, L. Lind, LPL, MC, MEK, MF, MM, MRI, OM, PSW, R. Sorice, SB, SKM, TES, TL, TN, TPC, TZ, UB, UV, and V. Großmann. Statistical analysis: AS, AT, CL, C. Minelli, C. Monnereau, C. Müller, DCS, D. Malzahn, D. Medenwald, DP, DR, DT, EH, FDGM, GFM, HL, JAB, JCB, JFF, JGS, JK, JNH, JS, KMR, LF, LPL, MC, MEK, MF, MGL, MHC, MM, MMN, MRI, NDD, PS, PSW, R. Sorice, SJS, SKM, S. Nuding, SP, and THP. Interpretation of data: ACM, ADH, AGU, AH, AJB, AS, ASPS, AT, A.V. Smith, A.V. Stanton, AZ, BHS, BP, CAM, DKA, EB, FR, GFM, HL, H. Schmidt, H. Schunkert, JFF, JGS, JM, JNH, JWD, KL, LF, LJL, MD, MF, MGL, MJGL, ML, MM, NS, OHF, OM, PMN, PS, PSW, R. Schmidt, RSV, SBF, SJS, ST, TBH, THP, TPC, TZ, V. Gudnason, VWVJ, WL, and WM. Drafting the manuscript: AS, AT, JFF, MD, PSW, RSV, and TZ. Revising manuscript critically for important intellectual content: all authors.

\section{Funding summary and Acknowledgments}

This work was supported by a grant from the US National Heart, Lung, and Blood Institute (N01-HL-25195; R01HL 093328 to RSV), a MAIFOR grant from the University Medical Center Mainz, Germany (to PSW), the Center for Translational Vascular Biology (CTVB) of the Johannes Gutenberg-University of Mainz, and the Federal Ministry of Research and Education, Germany (BMBF 01EO1003 to PSW). This work was also supported by the research project Greifswald Approach to Individualized Medicine (GANI_MED). GANI_MED was funded by the Federal Ministry of Education and Research and the Ministry of Cultural Affairs of the Federal State of Mecklenburg, West Pomerania (contract 03IS2061A). We thank all study participants, and the colleagues and coworkers from all cohorts and sites who were involved in the generation of data or in the analysis. We especially thank Andrew Johnson (FHS) for generation of the gene annotation database used for analysis. We thank the German Center for Cardiovascular Research (DZHK e.V.) for supporting the analysis and publication of this project. RSV is a member of the Scientific Advisory Board of the DZHK. Data on CAD and MI were contributed by CARDIoGRAMplusC4D investigators. See Supplemental Acknowledgments for consortium details. PSW, JFF, AS, AT, TZ, RSV, and MD had full access to all of the data in the study and take responsibility for the integrity of the data and the accuracy of the data analysis.

\section{Study-specific Acknowledgments, funding, and ethics statements}

The following list provides the study-specific names and funding sources with grant numbers only. Details on the study acronyms, study-specific Acknowledgments, funding, support for researchers, and ethics statements are provided in the supplemental material.

\section{AortaGen}

AGES. NIH N01-AG-1-2100, National Institute on Aging (NIA) Intramural Research Program, Hjartavernd (the Icelandic Heart Association), Althingi (the Icelandic Parliament).

ASCOT. Pfizer, New York; Servier Research Group, Paris, and Leo Laboratories, Copenhagen; partial funding: Barts and the London School of Medicine and Dentistry, Centre Nationale de Genotypage Paris, and Irish Research Council GREP award. 
ASPS. Austrian Science Fund Project P20545_P05 Genetics of Cerebral Small Vessel Disease.

CARDIA. National Heart, Lung, and Blood Institute in collaboration with the University of Alabama at Birmingham (HHSN268201300025C and HHSN268201300026C), Northwestern University (HHSN268201300027C), University of Minnesota (HHSN268201300028C), Kaiser Foundation Research Institute (HHSN268201300029C), and Johns Hopkins University School of Medicine (HHSN268200900041C); partial funding: Intramural Research Program of the NIA, Gene Environment Association Studies (GENEVA) through grants U01-HG004729, U01-HG04424, and U01HG004446 from the National Human Genome Research Institute.

CARLA. Deutsche Forschungsgemeinschaft (DFG) as part of Collaborative Research Center 598 "Heart Failure in the Elderly Cellular Mechanisms and Therapy," Medical Faculty, Martin-LutherUniversity Halle-Wittenberg; Wilhelm-Roux Programme, MartinLuther-University Halle-Wittenberg; Federal Employment Office; and Ministry of Education and Cultural Affairs of Saxony-Anhalt.

\section{CHARGE-HF}

CHS. National Heart, Lung, and Blood Institute (NHLBI) HHSN268201200036C, HHSN268200800007C, NO1HC55222, N01HC85079, N01HC85080, N01HC85081, N01HC85082, N01HC85083, N01HC85086, N01HC85084, N01HC35129; and NHLBI grants U01HL080295, R01HL087652, R01HL105756, R01HL103612, R01HL120393, and HL130114, with additional contribution from the National Institute of Neurological Disorders and Stroke (NINDS), and R01AG023629 from the NIA; partial funding: National Center for Advancing Translational Sciences, CTSI grant UL1TR000124, and National Institute of Diabetes and Digestive and Kidney Disease Diabetes Research Center (DRC) grant DK063491 to the Southern California Diabetes Endocrinology Research Center.

Cilento. Italian Ministry of Universities and CNR (Interomics Flagship Project, PONO3PE_00060_7), Assessorato Ricerca Regione Campania, Fondazione con il SUD (2011-PDR-13), and Istituto Banco di Napoli - Fondazione to MC.

DEPICT. Danish Council for Independent Research Medical Sciences (FSS), Alfred Benzon Foundation.

FHS1, FHS2, FHS3. National Heart, Lung and Blood Institute's Framingham Heart Study (NO1-HC-25195 and HHSN268201500001I), research grants (5R01HL107385-04, 1R01HL126136-01A1, R00HL107642, R01HL131532), Affymetrix Inc. for genotyping services (contract NO2-HL-6-4278).

Generation R. Erasmus Medical Center, Rotterdam, Erasmus University Rotterdam, Netherlands Organization for Health Research and Development (ZonMw), Netherlands Organisation for Scientific Research (NWO), Ministry of Health, Welfare and Sport and the Ministry of Youth and Families.

GHS-I, GHS-II, GHS-III. Government of Rhineland-Palatinate (“Stiftung Rheinland-Pfalzfür Innovation,” contractAZ961-386261/733), research programs "Wissen schafft Zukunft" and "Center for Translational Vascular Biology (CTVB)" of the Johannes Gutenberg-University of Mainz, Boehringer Ingelheim, and PHILIPS Medical Systems.

HyperGEN. HyperGEN echocardiography ancillary study: NIH (R01 HL 55673). HyperGEN parent study: Cooperative agreements (U10) with NHLBI: HL54471, HL54472, HL54473, HL54495, HL54496, HL54497, HL54509, HL54515.
JHS. NHLBI and National Institute on Minority Health and Health Disparities (NIMHD) (HSN268201300046C, HHSN268201300047C, HHSN268201300048C, HHSN268201300049C, HHSN268201300050C).

KNHI. National Genome Research Network (NGFN), German Federal Ministry of Education and Research (BMBF; 01GS0837 and 01GS0422); Competence Network for Heart Failure Germany.

KORA-F3 and KORA-F4. Helmholtz Zentrum München, German Research Center for Environmental Health, Neuherberg, Germany, BMBF; partial funding: NGFN.

LURIC. European Union 7th Framework Program (AtheroRemo, grant agreement 201668, and RiskyCAD, grant agreement 305739), INTERREG-IV-Oberrhein-Program (project A28, Genetic Mechanisms of Cardiovascular Diseases), European Regional Development Fund (ERDF), Wissenschaftsoffensive TMO.

MAGNet. NIH R01HL105993.

MICROS. Ministry of Health and Department of Educational Assistance, University and Research of the Autonomous Province of Bolzano, the South Tyrolean Sparkasse Foundation, and European Union framework program 6 EUROSPAN project (contract LSHGCT-2006-018947).

MPP. Swedish Heart-Lung Foundation, Swedish Research Council, European Research Council, Faculty of Medicine of Lund University, Skåne University Hospital, and the Crafoord Foundation.

NOMAS. NINDS, NIH (R37 NS2993 and R01 NS36286).

PIVUS. Swedish Foundation for Strategic Research (project grant ICA08-0047), Swedish Research Council (project grant 2012-1397), Swedish Heart-Lung Foundation (project grant 20120197), Swedish Society of Medicine, and Uppsala University.

RS-I, RS-II, RS-III. Netherlands Organisation of Scientific Research (NWO) investments (no. 175.010.2005.011, 911-03-012), Research Institute for Diseases in the Elderly (014-93-015; RIDE2), Netherlands Genomics Initiative (NGI)/NWO project 050-060-810, Erasmus MC and Erasmus University, Rotterdam, Netherlands Organization for Health Research and Development (ZonMw), Research Institute for Diseases in the Elderly (RIDE), Ministry of Education, Culture and Science, Ministry for Health, Welfare and Sport, European Commission (DG XII), and Municipality of Rotterdam. Partial funding: NWO investments (no. 175.010.2005.011, 911-03-012), Research Institute for Diseases in the Elderly (014-93-015; RIDE2), NGI/NWO project 050-060-810.

SHIP, SHIP-Trend. Federal Ministry of Education and Research (grants 01ZZ9603, 01ZZ0103, and 01ZZ0403), Ministry of Cultural Affairs, Social Ministry of the Federal State of Mecklenburg-West Pomerania. Partial funding: Competence Network Heart Failure of the Federal Ministry of Education and Research, Federal Ministry of Education and Research (grant 03ZIK012) and a joint grant from Siemens Healthcare, Erlangen, Germany and the Federal State of Mecklenburg-West Pomerania.

ULSAM. Swedish Foundation for Strategic Research (project grant no. ICA08-0047), Swedish Research Council (project grant 20121397), Swedish Heart-Lung Foundation (project grant 20120197), Swedish Society of Medicine, and Uppsala University; partial funding: SNP\&SEQ Technology Platform in Uppsala, Uppsala University, Uppsala University Hospital, Science for Life Laboratory-Uppsala, and the Swedish Research Council (contracts 80576801 and 70374401).

YFS. Academy of Finland: grants 286284, 134309 (Eye), 126925, 121584, 124282, 129378 (Salve), 117787 (Gendi), and 41071 (Skidi); the 
Social Insurance Institution of Finland; Competitive State Research Financing of the Expert Responsibility area of Kuopio, Tampere, and Turku University Hospitals (grant X51001); Juho Vainio Foundation; Paavo Nurmi Foundation; Finnish Foundation for Cardiovascular Research; Finnish Cultural Foundation; Tampere Tuberculosis Foundation; Emil Aaltonen Foundation; Yrjö Jahnsson Foundation; Signe and Ane Gyllenberg Foundation; and Diabetes Research Foundation of the Finnish Diabetes Association.

\section{Role of the sponsors}

The sponsors had no role in the study design, analyses, drafting of the manuscript, or the decision to publish.

Address correspondence to: Philipp S. Wild, Center for Cardiology and Center for Thrombosis and Hemostasis, University Medical
Center of the Johannes Gutenberg-University Mainz, Langenbeckstr. 1, 55131 Mainz, Germany. Phone: 49.6131.17.7163; E-mail: philipp.wild@unimedizin-mainz.de. Or to: Janine F. Felix, Department of Epidemiology, room Na-2906, Erasmus MC, University Medical Center Rotterdam, P.O. Box 2040, 3000 CA Rotterdam, Netherlands. Phone: 31.10.70.43997; E-mail: j.felix@erasmusmc. nl. Or to: Ramachandran S. Vasan, Sections of Preventive Medicine and Epidemiology and Cardiology, Department of Medicine, Boston University School of Medicine, and the Framingham Heart Study, 801 Massachusetts Avenue, Suite 470, Boston, Massachusetts 02118, USA. Phone: 617.638.8080; E-mail: vasan@ bu.edu. Or to: Marcus Dörr, Department of Internal Medicine B, University Medicine Greifswald, Ferdinand-Sauerbruch Str., 17475 Greifswald, Germany. Phone: 49.3834.86.80510; E-mail: mdoerr@uni-greifswald.de.
1. Go AS, et al. Executive summary: heart disease and stroke statistics - 2014 update: a report from the American Heart Association. Circulation. 2014;129(3):399-410.

2. Vasan RS, et al. Genetic variants associated with cardiac structure and function: a meta-analysis and replication of genome-wide association data. JAMA. 2009;302(2):168-178.

3. Smith NL, et al. Association of genome-wide variation with the risk of incident heart failure in adults of European and African ancestry: a prospective meta-analysis from the cohorts for heart and aging research in genomic epidemiology (CHARGE) consortium. Circ Cardiovasc Genet. 2010;3(3):256-266.

4. Vasan RS, et al. Genome-wide association of echocardiographic dimensions, brachial artery endothelial function and treadmill exercise responses in the Framingham Heart Study. BMC Med Genet. 2007;8(suppl 1):S2.

5. Lee DS, et al. Association of parental heart failure with risk of heart failure in offspring. $N$ Engl J Med. 2006;355(2):138-147.

6. Lee DS, et al. Relation of disease pathogenesis and risk factors to heart failure with preserved or reduced ejection fraction: insights from the framingham heart study of the national heart, lung, and blood institute. Circulation. 2009;119(24):3070-3077.

7. Ho JE, et al. Discriminating clinical features of heart failure with preserved vs. reduced ejection fraction in the community. Eur Heart J. 2012;33(14):1734-1741.

8. Ho JE, et al. Predictors of new-onset heart failure: differences in preserved versus reduced ejection fraction. Circ Heart Fail. 2013;6(2):279-286.

9. Andersson C, Vasan RS. Epidemiology of heart failure with preserved ejection fraction. Heart Fail Clin. 2014;10(3):377-388.

10. Bulik-Sullivan BK, et al. LD Score regression distinguishes confounding from polygenicity in genome-wide association studies. Nat Genet. 2015;47(3):291-295.

11. Skol AD, Scott LJ, Abecasis GR, Boehnke M. Joint analysis is more efficient than replication-based analysis for two-stage genome-wide association studies. Nat Genet. 2006;38(2):209-213.

12. Jaddoe VW, et al. The Generation R Study: design and cohort update 2012. Eur J Epidemiol. 2012;27(9):739-756.

13. Vasan RS, Larson MG, Benjamin EJ, Evans JC, Levy D. Left ventricular dilatation and the risk of congestive heart failure in people without myocardial infarction. N Engl J Med. 1997;336(19):1350-1355.

14. Saneyoshi T, Kume S, Amasaki Y, Mikoshiba K. The Wnt/calcium pathway activates NF-AT and promotes ventral cell fate in Xenopus embryos. Nature. 2002;417(6886):295-299.

15. Dejmek J, Safholm A, Kamp Nielsen C, Andersson $\mathrm{T}$, Leandersson $\mathrm{K}$. Wnt-5a/Ca ${ }^{2+}$-induced NFAT activity is counteracted by Wnt-5a/ Yes-Cdc42-casein kinase $1 \alpha$ signaling in human mammary epithelial cells. Mol Cell Biol. 2006;26(16):6024-6036.

16. Heineke J, Molkentin JD. Regulation of cardiac hypertrophy by intracellular signalling pathways. Nat Rev Mol Cell Biol. 2006;7(8):589-600.

17. Encode Project Consortium. A user's guide to the encyclopedia of DNA elements (ENCODE). PLoS Biol. 2011;9(4):e1001046.

18. Rossin EJ, et al. Proteins encoded in genomic regions associated with immune-mediated disease physically interact and suggest underlying biology. PLoS Genet. 2011;7(1):e1001273.

19. Pers TH, et al. Biological interpretation of genome-wide association studies using predicted gene functions. Nat Commun. 2015;6:5890.

20. Lee YG, Macoska JA, Korenchuk S, Pienta KJ. MIM, a potential metastasis suppressor gene in bladder cancer. Neoplasia. 2002;4(4):291-294.

21. Saarikangas J, et al. Missing-in-metastasis MIM/ MTSS1 promotes actin assembly at intercellular junctions and is required for integrity of kidney epithelia. JCell Sci. 2011;124(pt 8):1245-1255.

22. Ikram MK, et al. Four novel loci (19q13, 6q24, 12q24, and 5q14) influence the microcirculation in vivo. PLoS Genet. 2010;6(10):e1001184.

23. Levy D, et al. Genome-wide association study of blood pressure and hypertension. Nat Genet. 2009;41(6):677-687.

24. International Consortium for Blood Pressure Genome-Wide Association Studies, et al. Genetic variants in novel pathways influence blood pressure and cardiovascular disease risk. Nature. 2011;478(7367):103-109.
25. Newton-Cheh C, et al. Genome-wide association study identifies eight loci associated with blood pressure. Nat Genet. 2009;41(6):666-676.

26. Schunkert H, et al. Large-scale association analysis identifies 13 new susceptibility loci for coronary artery disease. Nat Genet. 2011;43(4):333-338.

27. Pan S, et al. A haplotype of the GOSR2 gene is associated with myocardial infarction in Japanese men. Genet Test Mol Biomarkers. 2013;17(6):481-488.

28. Beitelshees AL, et al. CACNA1C gene polymorphisms, cardiovascular disease outcomes, and treatment response. Circ Cardiovasc Genet. 2009;2(4):362-370.

29. Gertow K, et al. Identification of the BCAR1CFDP1-TMEM170A locus as a determinant of carotid intima-media thickness and coronary artery disease risk. Circ Cardiovasc Genet. 2012;5(6):656-665.

30. Sabater-Lleal M, et al. Common genetic determinants of lung function, subclinical atherosclerosis and risk of coronary artery disease. PLoS One. 2014;9(8):e104082.

31. Hu B, Copeland NG, Gilbert DJ, Jenkins NA, Kilimann MW. The paralemmin protein family: identification of paralemmin-2, an isoform differentially spliced to AKAP2/AKAP-KL, and of palmdelphin, a more distant cytosolic relative. Biochem Biophys Res Commun. 2001;285(5):1369-1376.

32. Ivanov DV, et al. A new human gene KCNRG encoding potassium channel regulating protein is a cancer suppressor gene candidate located in 13q14.3. FEBS Lett. 2003;539(1-3):156-160.

33. Dunckley T, et al. Whole-genome analysis of sporadic amyotrophic lateral sclerosis. $N$ Engl J Med. 2007;357(8):775-788.

34. Ousingsawat J, Kongsuphol P, Schreiber R, Kunzelmann K. CFTR and TMEM16A are separate but functionally related $\mathrm{Cl}^{-}$channels. Cell Physiol Biochem. 2011;28(4):715-724.

35. Malekar P, et al. Wnt signaling is critical for maladaptive cardiac hypertrophy and accelerates myocardial remodeling. Hypertension. 2010;55(4):939-945.

36. Fan Q, et al. Inhibition of Fas-associated death domain-containing protein (FADD) protects against myocardial ischemia/reperfusion injury in a heart failure mouse model. PLoS One. 2013;8(9):e73537. 


\section{CLINICAL MEDICINE}

37. Shah SJ, Katz DH, Deo RC. Phenotypic spectrum of heart failure with preserved ejection fraction. Heart Fail Clin. 2014;10(3):407-418.

38. Gabriel RS, Klein AL. Modern evaluation of left ventricular diastolic function using Doppler echocardiography. Curr Cardiol Rep. 2009;11(3):231-238.

39. Sohn DW. Heart failure due to abnormal filling function of the heart. J Cardiol. 2011;57(2):148-159.

40. Oh JK, Appleton CP, Hatle LK, Nishimura RA, Seward JB, Tajik AJ. The noninvasive assessment of left ventricular diastolic function with twodimensional and Doppler echocardiography. JAm Soc Echocardiogr. 1997;10(3):246-270.

41. Redfield MM, Jacobsen SJ, Burnett JC, Mahoney DW, Bailey KR, Rodeheffer RJ. Burden of systolic and diastolic ventricular dysfunction in the community: appreciating the scope of the heart failure epidemic. JAMA. 2003;289(2):194-202.

42. Lang RM, et al. Recommendations for chamber quantification. Eur J Echocardiogr. 2006;7(2):79-108.

43. Willer CJ, Li Y, Abecasis GR. METAL: fast and efficient meta-analysis of genomewide association scans. Bioinformatics. 2010;26(17):2190-2191.

44. Purcell S, et al. PLINK: a tool set for whole-genome association and population-based linkage analyses. Am J Hum Genet. 2007; 81(3):559-575.

45. Mitchell GF, et al. Common genetic variation in the 3'-BCL11B gene desert is associated with carotid-femoral pulse wave velocity and excess cardiovascular disease risk: the AortaGen Consortium. Circ Cardiovasc Genet. 2012;5(1):81-90.

46. Redheuil A, et al. Age-related changes in aortic arch geometry: relationship with proximal aortic function and left ventricular mass and remodel- ing. J Am Coll Cardiol. 2011;58(12):1262-1270.

47. Nikpay M, et al. A comprehensive 1,000 Genomes-based genome-wide association meta-analysis of coronary artery disease. Nat Genet. 2015;47(10):1121-1130.

48. Yang J, Lee SH, Goddard ME, Visscher PM. GCTA: a tool for genome-wide complex trait analysis. Am J Hum Genet. 2011;88(1):76-82.

49. Westra HJ, et al. Systematic identification of trans eQTLs as putative drivers of known disease associations. Nat Genet. 2013;45(10):1238-1243.

50. Zeller T, et al. Genetics and beyond - the transcriptome of human monocytes and disease susceptibility. PLoS One. 2010;5(5):e10693.

51. Pe'er I, Yelensky R, Altshuler D, Daly MJ. Estimation of the multiple testing burden for genomewide association studies of nearly all common variants. Genet Epidemiol. 2008;32(4):381-385. 